\title{
Mobile gaze tracking and an extended linkography for collaborative sketching and designing
}

\author{
Tellervo Härkki ${ }^{1}$ iD
}

Accepted: 13 January 2022 / Published online: 15 February 2022

(c) The Author(s) 2022

\begin{abstract}
The demand for teaching and learning collaborative design is probably greater than ever thanks to many influential parties highlighting creativity, collaboration and designing as necessary future skills. However, 'good' collaborative design necessitates a clear understanding of what constitutes 'good' and 'collaboration', and of how an individual's activities contribute to a team achieving a satisfactory design solution. This research aimed to develop a methodological approach to facilitate the analysis of collaborative interaction and design as equally meaningful and closely linked components, and to equally account for verbal and sketching activities. The developed extended linkography accounts for verbal and sketched design moves and utilises mobile gaze-tracker data to confirm visual attention and linkages between sketches. The method has potential importance for research on design cognition and collaboration, but also for analysing collaborative problem solving in other contexts. To illustrate the method, a case study of packaging design students collaboratively designing a sustainable Christmas basket is presented. The gaze data revealed that students' collaborative engagement with 'shared' sketches differed: from the two proposals selected for 3D mock-up-building, one was developed concertedly and one as an aside of the shared process. The latter development appeared to be independent, seeking little input from team members. All in all, three qualitatively different interactive design processes were identified, and quantitative levels of collaboration were measured through Convergent and Divergent Collaboration Indexes. To determine the role of sketches for each of the students' designing, gaze data was indispensable.
\end{abstract}

Keywords Collaboration · Convergent collaboration · Divergent collaboration · Design education $\cdot$ Linkography $\cdot$ Mobile gaze tracking $\cdot$ Sketching

\section{Introduction}

The need for teaching and learning collaboration, design and creativity is probably greater than ever, as several influential parties have continued to promote these skills as necessary for future success (Binkley et al., 2012; Dunn et al., 2017; for a review, see Dede, 2009). A

Tellervo Härkki

tellervo.harkki@helsinki.fi

1 University of Helsinki, Helsinki, Finland 
precondition for such teaching is understanding what constitutes constructive and effective collaboration (Kiernan et al., 2020); however, the discussion on this topic has yet to reach a consensus. Currently, the relationship between efficient collaboration and good design also remains unclear (Safin et al., 2019).

Achieving a better understanding regarding these topics requires recognising the difference between collaborative situations and collaborative interactions. Dillenbourg (1999) characterised the difference as follows. In a collaborative situation, participants have a common goal, work together, are more or less at the same level and can perform the same actions. However, collaborative interaction involves interactivity and synchronicity - specific types of activities. Interactivity refers to the influence that interactions have on other participants' cognitive processes, not to the frequency of those interactions. Synchronicity does not refer to a technology, but to a considerate meta-communicative contract: Partners expect each other to wait for their messages and they process the message content as soon as it is delivered (Dillenbourg, 1999). Organising students to work in teams creates the possibility for collaboration, but does not guarantee collaboration. Research on collaborative design has often focused on design cognition in collaborative situations, but not always on collaborative interactions. Likewise, not much attention has been paid to the specifics of collaborative interaction in design (Hultén et al., 2018; Reiter-Palmond \& Leone, 2018). A gap exists, as there is no theoretical basis that accounts for both halves of 'collaborative design'.

To complicate things even more, no unified definition of collaboration exists, and the word is in danger of becoming diluted (Tessier, 2020). For instance, Hennessy and Murphy $(1999$, p. 1) defined collaboration as 'pupils actively communicating and working together to produce a single outcome, talking and sharing their cognitive resources to establish joint goals and referents, to make joint decisions, to solve emerging problems, to construct and modify solutions and to evaluate the outcomes through dialogue and action.' Some have used collaboration interchangeably with co-operation (Tessier, 2020), while a clear distinction has been made, for example, by Roschelle and Teasley (1995), to whom collaboration refers to co-ordinated synchronous activity, and where co-operation takes place around a division of labour and responsibilities. This research adopts the definition by Hennessy and Murphy.

For collaborative design researchers, collaboration is not a homogeneous process. Kvan's (2000) review suggested that collaboration does not change the episodic and cyclical nature of design: Design is a series of discrete activities. Likewise, collaborative design entails a series of discrete steps with differing levels of sharedness; working together is interrupted by individual working, and participants address design issues from their own perspective (Kvan, 2000). Visser (2006) held a similar two-fold view on collaborative design. Essential parts of collaborative design advance through interaction between designers. This interaction involves the shared ideation and processing of ideas rather than mere externalisations of an individual's previously developed ideas; simultaneously, an individual's design activity has an important role in the whole process (Visser, 2006).

Despite a commendable volume of research on collaborative design since the 1990s, few methodological approaches have been widely embraced (Tang et al., 2011). Long-standing design research methodologies, such as Function-Behaviour-Structure (Gero, 1990) and linkography (Goldschmidt, 1995, 2014), do not address the specifics of collaborative interaction. These methods focus on design, even though they have also been applied to analyse collaboration. For instance, Goldschmidt (1995) calculated each designer's contributions, but suggested that interdependency is a more complicated issue that requires qualitative analysis. Measures separating ideas built on each other's ideas from ideas built on one's 
own ideas have been calculated (van der Lugt, 2005), as have the volumes of these two types of interactions per designer (Kan \& Gero, 2011). Yet, these measures do not characterise the process; thus, a methodological gap exists.

Another methodological gap relates to the role of sketching in collaboration. Despite the acknowledged importance of sketching (Hua, 2019; Purcell \& Gero, 1998) and its many uses in design (Brun et al., 2016; Eckert et al., 2012; Uziak \& Fang, 2018), the analysis of collaborative sketching often resorts to second-hand information. As research data, sketched visualisations can be replaced by verbal transcripts and protocols, and designers' visual attention has been observed rather than measured. A verbal protocol of collaborative design cannot account for silent processing that takes place simultaneously with speech. The availability of multiple stimuli (speech and sketches) increases the possibility of designers' activities being directed towards individual trails instead of following a shared process. These individual trails are difficult to observe accurately. The question is how to capture the silent activity of sketching and an individual's visual attention.

A potential game changer is provided by mobile gaze tracking. It is still rare in research on collaborative co-located design, but studies on collaborative learning have revealed linkages between speech, visual materials and the production of new ideas. A close relationship exists between an individual's gaze target and what that person is thinking about (Just \& Carpenter, 1980). The findings from collaborative problem solving include the role of the available visuals in overcoming a standstill and generating a productive new idea (Hannula, 2016) and the explorative role of gazing during long silent periods (Hannula \& Williams, 2016). These promising findings suggest that mobile gaze tracking could support better capturing the mechanisms behind the importance of sketching for design and creativity (Goel, 1995; Purcell \& Gero, 1998; van der Lugt, 2005) and understanding the value of collaborative sketching.

This understanding could be especially important for design education. Many researchers suggest that due to a lack of technical skills and - more importantly - due to a lack of understanding of sketching as an exploration tool, novices do not benefit from sketches and sketching as much as professional designers do (Booth et al., 2008; Casakin \& Goldschmidt, 1999; Deininger et al., 2017; Goldschmidt, 1991; Suwa \& Tversky, 1997; Ullman et al., 1990). While studies comparing design novices with experts exist, there is very little research on how novices can become experts, particularly within teams (Kiernan et al., 2020; Smith, 2015). Design education facilitates the transformation from a novice to a professional designer, and design educators are required to understand expert strategies (the targeted knowledge and skill), as well as novice performance. Understanding novices facilitates choosing suitable instructional strategies and directing (often limited) teaching resources purposefully. Otherwise, design educators risk offering misguided instructions. As Casakin and Goldschmidt (1999, p. 174) noted, 'Novice designers do not need to be taught how to use analogy: they already have this cognitive capacity. They do need, however, to be shown how and why it can be helpful to harness this ability for successful design problem-solving.'

While our long-term objective is to support collaborative design theory building as an interactive endeavour and to recognise productive interaction that supports design creativity, this explorative research developed a methodological approach to facilitate the analysis of collaborative interaction and design as equally meaningful and closely linked components. As this approach understands verbal and sketching activities as equally meaningful, the next section revisits research approaches to face-to-face collaborative design and sketching. 


\section{Theoretical background}

\section{Collaboration and interaction in collaborative design}

Interaction is elemental to collaborative designing and it influences the structure of the design activity. According to Goldschmidt (2014), collaborative design can appear to be more episodic and iterative than individual design, as each designer can go far back in time to his/her own previous ideas, despite the issues that are currently under discussion. In what follows, we set aside team process models (cf. Sauder \& Jin, 2016) that address a collaborative design team as a single actor instead of scrutinising individual participation and interaction. The emphasis herein is on diverse approaches to analyse co-located design collaboration; three approaches were detected.

First, activity-level research emphasises action sequences rather than particular types of actions. Kiernan et al. (2020) aimed at understanding how to support novice designers' collaboration skills (engaging in constructive dialogue). Their results emphasised the importance of the depth of exploration and of considering alternative perspectives. Resonating with Kleinsmann et al. (2012), who noted that the degree of knowledge sharing was central for collaborative success, as deeper reasoning facilitated knowledge integration, Kiernan et al.'s (2020) results combine collaborative activities with domain-specific knowledge. Le Bail et al. (2020) studied collaborative design dialogue in a situation where designers' core values were at stake. They recognised collaborative argumentation, in which designers openly explored arguments, aimed at collectively accepted agreement based on the evoked arguments and where they entertained no fixed personal commitments. In conflictual argumentation, designers determinedly defended their own views and refuted those of others (Le Bail et al., 2020). Barron (2003) connected neglecting others' views to low performance and failure (in collaborative problem solving). Corroborating this, Le Bail et al. (2020) found that when conflicts were not genuinely resolved, solutions were 'acceptable' rather than 'good'; they emphasised the need for resolving conflicts and for moderating activities.

Second, activities were combined with episodes by Wiltschnig et al. (2013), who studied collaborative exploration episodes (e.g. the co-evolution of a problem and solution, the exploration of solutions for a specific requirement, identifying a new requirement based on a solution idea and a solution attempt while considering particular requirements). They rated episodes that comprised speech acts by different individuals as collaborative and calculated the percentages of collaborative episodes from all episodes. However, they acknowledged that it was impossible to tally unspoken individual episodes; thus, the percentages were not all-inclusive. Darses et al. (2001) highlighted that collaborating teams not only produced design solutions but also the preconditions for reaching a satisfactory solution (goal setting, problem descriptions and justified evaluations that are frequently extended with alternative propositions). Thus, it is not just a single type of activity that matters but the way in which the activities are sequenced.

Third, repetitive patterns of designers' interaction were targeted by Sauder and Jin (2016). They combined types of collaborative stimulation and thinking processes. Their model assumes that in a collaborative context, designers' thinking processes are interactive rather than independent. Collaboratively shared resources inspire individuals' thought processes. Further, their results suggest that questioning can be more effective than the presentation of shared resources. 


\section{Sketching in collaborative design}

Visser (2006) conceptualised design as the construction of representations. Sketching and visual thinking have often been central features of descriptions of meaningful collaborative events (e.g. Cross \& Clayburn Cross, 1995; Murphy, 2012). Another approach has been to classify sketches. While detailed typologies illustrate the purposes of design sketches (see the review in Schembri et al., 2015), they fall short of recognising whether a single sketch serves multiple functions (van der Lugt, 2005) and of explaining the unfolding activities along the timeline.

Focusing on the complete design process, Schembri et al. (2015) suggested that sketches produced to share and explain an idea are crucial in promoting collaboration and decision-making. Two more detailed approaches recognised sketching-related patterns, yet of a very different character. By analysing speech, sketching and gesturing activities, Eris et al. (2014) identified a pattern in which new potential solutions emerged after previously unconnected problem- and solution-focused sketch elements were associated through cross-gesturing (gesturing at a sketch created by someone else). While targeting dynamic patterns in developing a shared understanding, Cash et al. (2020) combined activities (dealing with information, representations, or knowledge sharing) with taskwork (e.g. problem understanding, solution generation and solution decision-making) or teamwork (dealing with a process or communication, coordination and process decision-making). In their study, the development of a shared understanding was connected to two sketching-related patterns. First, relevant information is gathered and discussed while using conversational pauses to process and record co-constructed knowledge as representations. Second, team coordination is combined with representation activity at process turning points.

To conclude, models involving collaborating designers' verbal and sketched contributions that are based on activity sequences or patterns could better reflect the character of collaborative designing as a constant interaction between individuals and externalised representations, such as sketches. According to Goldschmidt (2011, p. 63), 'visual stimuli have been shown to have a considerable impact on design creativity, but their crucial role is not reflected in most current design creativity models.' While interaction is observable, capturing targets of an individual's visual attention and his/her internal thinking processes remains challenging. Leveraging mobile gaze tracking takes us one step closer to understanding the use of visual stimuli in collaborative design.

\section{Gaze tracking in collaborative design research}

Mobile gaze tracking has provided promising results in research on collaboration. The method could also advance design research, in which the use of static (computer-based) trackers is currently more commonplace. For instance, Sun et al. (2014) studied individuals' digital sketching with static trackers and found that longer fixations were related to creative segments (loops involving idea generation, idea expression and perceiving the resulting sketch) but not to other design activities. Fixations refer to periods of time (between tens of milliseconds and several seconds) in which the gaze remains (relatively) still, which implies that the individual is collecting information that he/she is paying attention to (Holmqvist et al., 2011). A longer fixation duration indicates more engagement or difficulties in extracting information (Rayner, 1998), whereas more fixations on a particular area indicate that the area is more important or noticeable (Poole \& Ball, 2005). 
In general, gaze and visual attention are closely linked (Hayhoe \& Ballard, 2014), yet fixation does not guarantee information uptake and processing. The relationship between gaze and cognition (Hayhoe \& Ballard, 2014) or intentions (Park et al., 2016) is not straightforward. Many established eye-tracking measures (cf. Holmqvist et al., 2011) have been developed for the purposes of laboratory experiments. These measures typically omit development and change over time. To research complicated processes 'in the wild', these established measures need to be adapted and combined with other data to provide insights. For instance, in a study by Huang (2017) on creative problem solving, a mean fixation duration that gradually increased throughout the process indicated impasses rather than efficient information processing. Similarly, a typical gaze-tracking measure for collaboration - joint visual attention - requires combining gaze information with spatial and verbal information to provide a more refined account of the interaction, as joint visual attention itself does not guarantee high-quality collaboration (Schneider et al., 2018). Currently, no established methods exist that utilise mobile gaze tracking in research on collaborative co-located design. This research has chosen to build on linkography (Goldschmidt, 1995, 2014), an acknowledged design process research methodology that accommodates both the analysis of individual and collaborative design (Goldschmidt, 1995), is based on elements (links) that manifest interaction, and most importantly, that facilitates the integration of gaze and sketching data into verbal protocols.

\section{Research questions}

From these premises, collaborative design is understood as creative interaction translating into verbalisations, sketching and gazing. A new methodological approach was developed to facilitate the analysis of collaborative interaction and design as equally meaningful and closely linked components. Further, two research questions were set:

(1) What types of interactive design processes can be identified?

(2) What does gaze data reveal about the role of sketches in these processes?

The paper is structured as follows. The next section presents the methodological choices based on the theoretical outlines. Then, the Results section features the interactive design processes but also participants' visual attention placed on each other's sketches from two methodological vantage points: first, gaze data supporting the analysis of interactivity in design and second, more 'traditional' eye-tracking measures derived from the gaze data. Finally, the Discussion and Conclusions sections evaluate and synthesise our methodological insights with empirical findings, and consolidate a theoretical construct of intense collaborative design.

\section{Methodology}

\section{Linkography}

Linkography (Goldschmidt, 2014) represents design thinking as a network of interlinked segments of a verbal design process protocol. The methodology is especially powerful at indicating so-called significant moves, which are influential statements that either provide 
inspiration to multiple other moves or consolidate several previous moves into one (Goldschmidt, 2014). These presentations render visible the fine-grained origins of the developed concepts and the levels of the evaluation, refinement and synthesis activities that are involved. For collaborative design, this level of involvement with a certain idea could also be understood as a measure of active engagement between the collaborating designers - an indicator of the level of collaboration. The basic structure of linkography facilitates the integration of additional modalities (sketching and gaze) and maintains the chronological order of activities. Further, linkography allows the level of granularity to be adjusted according to the needs of the particular research (Hatcher et al., 2018).

Traditional linkography is based on verbal protocols parsed to design moves. A design move is considered to transform the situation somewhat in relation to the prior situation (Goldschmidt, 2014). Moves can be based on turn taking in speech, or each sentence can constitute a move - the most suitable level of granularity depends on the protocol and research aims. Interconnected moves are linked together, and when presented graphically as a linkograph, these links form network-like patterns (see the example in Fig. 1). In other words, links represent continuing lines of thought, such as evaluating a proposed feature. Moves that have a high number of links are considered critical moves (CMs) that have special importance for the process. Each link can be interpreted as a backlink (linking move $\mathrm{B}$ to a previous move $\mathrm{A}$, 'B building on/answering to $\mathrm{A}$ ') or as a forelink (linking move A to a later move B, 'A inspiring/prompting B'). Linkography also provides other analytical concepts based on links, such as network patterns (webs, chunks, sawtooth) and quantifications (link density, link index, link span). These concepts were not used in this research, but detailed descriptions and examples can be found in Goldschmidt (2014).

The assumption is that backlinks indicate convergent thinking and forelinks divergent thinking, both of which are necessary for designing (Goldschmidt, 1995, 2016). Moves with both backlinks and forelinks imply flexible shifts between divergent and convergent thinking - a strategy related to efficiency and effectiveness in design (Goldschmidt, 2014), as the variation between divergent and convergent approaches pushes the process forward (Kälviäinen \& Nylander, 2019).

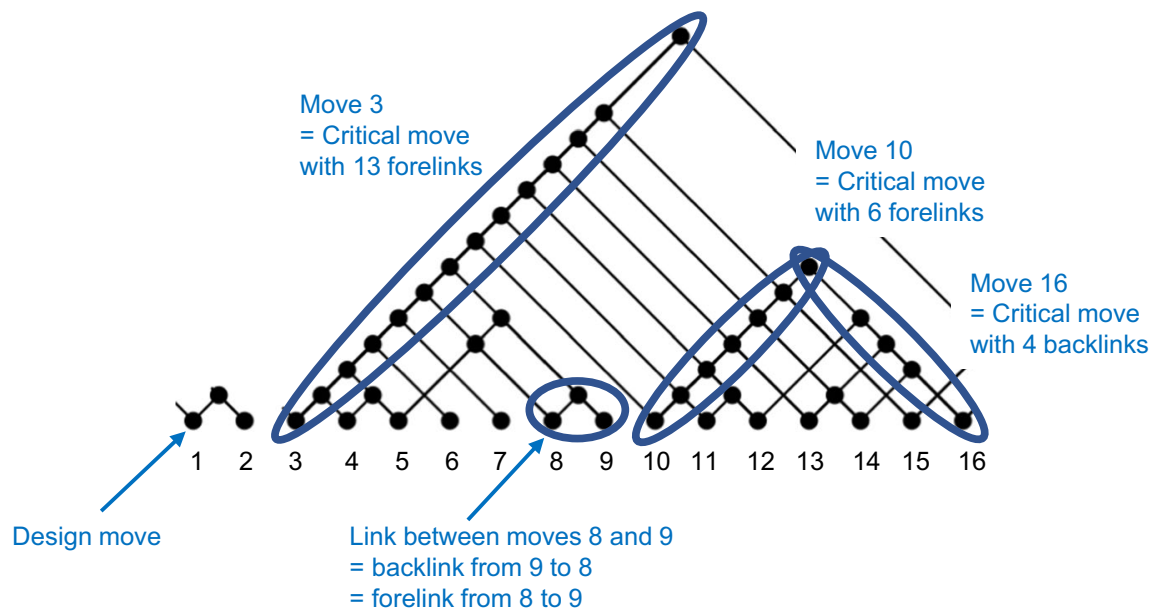

Fig. 1 An example visualising the basic terminology of linkographs: design move, backlink and forelink, critical moves. (In this case, the threshold number of links for a critical move was set to 4) 


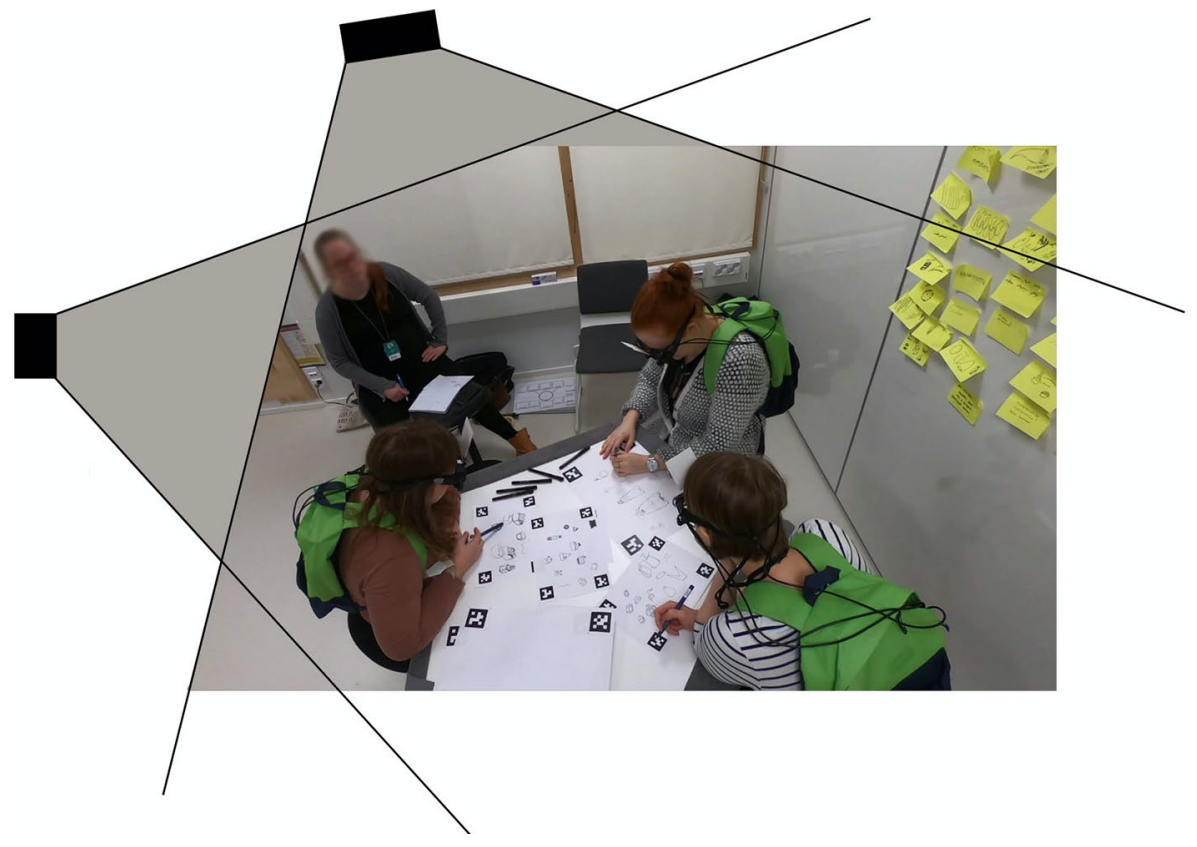

Fig. 2 Packaging design students designing sustainable Christmas baskets. The green backpacks are required to carry the laptops for gaze-data processing

A recent review on linkography is available in Blom and Bogaers (2020) and a review on adaptations is in Hatcher et al. (2018). Linkography adaptations by Cai et al. (2010) and van der Lugt (2005) identified sketches as design moves, but to the best of our knowledge, no adaptation combining different modalities (verbalisations, sketches etc.) has yet been published. Some adaptations centre on collaboration. van der Lugt (2005) distinguished between an 'individual' link (a link between moves by the same designer) and a 'shared' link (a link between moves by different designers). Similarly, Kan and Gero (2011) separated intra- and interpersonal design processes. An intrapersonal design process refers to an individual continuing his/her own line of thought, while an interpersonal design process refers to participants actively engaging in analysing, evaluating and building on each other's suggestions. Kan and Gero (2011) calculated the volumes of these links per participant. To conclude, linkography adaptations measure collaboration at the link and participant level, but, to date, not at the process level.

\section{Research setting}

The research was conducted at the packaging design department of LAB University of Applied Sciences, Finland. The setting (Fig. 2) involved a design brief, a group of three design students, their teacher (who was observing), one stationary video camera on a 
tripod and one top-view camera, as well as mobile gaze-tracking equipment ${ }^{1}$ that included spectacle-like gaze trackers with scene cameras and a connected laptop in a backpack.

\section{Participants}

Three design students participated in the research. These students were the first to answer their design lecturer's call for volunteers for a gaze-tracking study by the University of Helsinki, and they knew each other prior to this research. In the initial questionnaire (Appendix 1), Lisa and $\mathrm{Bea}^{2}$ (second-year packaging design students) claimed to have no professional design experience, and Rita (a third-year packaging design student) reported having less than one year of professional design experience. ${ }^{3}$ The students received a movie ticket (value 12.5 euros) in return for their participation. The students signed an informed consent form, and all procedures performed were in accordance with ethical principles of research with human participants in the human sciences in Finland (Finnish National Board of Research Integrity TENK Guidelines, 2019).

\section{Design challenge and data collection}

The details of the design challenge, tasks and collected data are available in Fig. 3.

\begin{tabular}{|c|c|c|c|c|c|c|}
\hline \multicolumn{7}{|c|}{ Preparations prior to the actual design task } \\
\hline $\begin{array}{l}\text { Introductions } \\
\text { Research information and consent form } \\
\text { introduced to students } \\
\text { Students sign consent forms } \\
\text { Students fill in intial questinnaire }\end{array}$ & $\begin{array}{l}\text { Calibrating the eye- } \\
\text { tracking equipment for } \\
\text { each student }\end{array}$ & \multicolumn{5}{|c|}{$\begin{array}{l}\text { Students got to familiriaze themselves with the eye-tracking equipment, sketching and collaboration with a } 10 \text {-minute "Year } \\
2040 \text { gift shop" themed warm-up task Sketch and pass over: } \\
\text { Sketch a gift shop in the year } 2040 \text {. After } 30 \text { seconds you'll hear a sound: please give your sketch sheet to the person sittin } \\
\text { on your right, who will continue the sketch you started. Yuu'll continue the sketch started by the person sitting on your left. } \\
\text { After } 6 \text { minutes, all sketching should stop. You'll get } 1 \text { minute to describe the gift shop in the sheet in front of you. }\end{array}$} \\
\hline \multicolumn{7}{|l|}{ Design challenge structure and timeline } \\
\hline $\begin{array}{l}\text { Design challenge and task structure is } \\
\text { explained to students. }\end{array}$ & \multicolumn{6}{|c|}{$\begin{array}{l}\text { Sustainable Christmas basket } \\
\text { During Christmastime, a considerable number of gourmet gift baskets of different kinds are sold. Afterwards } \\
\text { shapes and sizes are unpractical and often don't provide for any other re-use than packaging gifts. If the bas } \\
\text { to store for later use, especially when closet space at home is limited. Baskets given as business gifts can be } \\
\text { influential individuals receive multiple baskets every Christmas. A need exists for a modern version of a bask } \\
\text { sustainable yet appealing and festive. } \\
\text { The modern Christmas basket should be as eco-friendly as possible and its appealing look should be based } \\
\text { colours. While serving as a package and removing the need to be topped with plastic wrapping, this modern } \\
\text { with ease. In addition to the end-user perspective, logistics such as cube utilization and enablement of efficie } \\
\text { Task 1: Ideate a gourmet basket. Use sticky notes and the wall. } \\
\text { Task 2: Sketch your basket up to the point where building a 3D model is feasible. Use A4 and A3 sheets. } \\
\text { Task 3: Build a 3D cardboard mock-up (i.e. non-functional model that shows the 3D shape and structure) }\end{array}$} \\
\hline Task & Task 1 & Task 2 & $\begin{array}{l}\text { Selection of a } \\
\text { solution }\end{array}$ & Task 3 & Break & Group interview \\
\hline $\begin{array}{l}\text { Time spent } \\
\text { (students could work as long as they } \\
\text { wanted) }\end{array}$ & 38 minutes & 35 minutes & 5 minutes & 85 minutes & $\begin{array}{l}45 \text { minutes } \\
\text { (lunch) }\end{array}$ & $\begin{array}{l}\text { Interrupted after } 25 \\
\text { minutes }\end{array}$ \\
\hline \multicolumn{7}{|c|}{ Process outcomes and collected research data } \\
\hline Student work & $\begin{array}{l}35 \text { sticky notes with } \\
\text { total } 20 \text { verbal ideas and } \\
37 \text { sketches }\end{array}$ & $\begin{array}{l}3 \text { A4 sheets and } 3 \text { A3 } \\
\text { sheets with } \\
\text { a total } 100 \text { sketches }\end{array}$ & $\begin{array}{l}2 \text { solutions selected } \\
\text { (Rita's and Lisa's) }\end{array}$ & $\begin{array}{l}\text { One mockup (Rita's) } \\
\text { (Lisa's solution proved } \\
\text { too difficult to build) }\end{array}$ & - & $\begin{array}{l}\text { Answering questions } \\
\text { while viewing selected } \\
\text { parts of gaze-overlaid } \\
\text { video }\end{array}$ \\
\hline $\begin{array}{l}\text { Other collected data } \\
\text { - horizontal and top-view video, total } \\
\text { - gaze video, total }\end{array}$ & $\begin{array}{l}76 \text { minutes } \\
114 \text { minutes }\end{array}$ & $\begin{array}{l}70 \text { minutes } \\
105 \text { minutes }\end{array}$ & $\begin{array}{l}10 \text { minutes } \\
15 \text { minutes }\end{array}$ & $\begin{array}{l}170 \text { minutes } \\
255 \text { minutes }\end{array}$ & & $\begin{array}{l}30 \text { minutes of } \\
\text { horizontal video data }\end{array}$ \\
\hline
\end{tabular}

Fig. 3 Design challenge and task structure, structure and collected research data. Only the data collected from the sketching task (Task 2, related data highlighted in bold) was utilised for this study. The initial questionnaire is available in Appendix 1

\footnotetext{
${ }^{1}$ The technical details of the utilised high-performance, non-commercial mobile gaze-tracking equipment are available in Toivanen et al. (2017). According to Toivanen et al. (2017), the equipment outperfomed (at the time) a best-in-class commercial device, the SMI system (with iView 2.1 recording system and BeGaze 3.5 analysing software) for spatial accuracy and precision. Instructions on building this tracker from standard off-theshelf components and a 3D printed frame are available in Lukander et al. (2013). The tracker software is available at https://www.brainworklab.fi/gazetracker/

2 All names are pseudonyms.

${ }^{3}$ Later, she explained that this experience included two commissions, each of less than two weeks.
} 

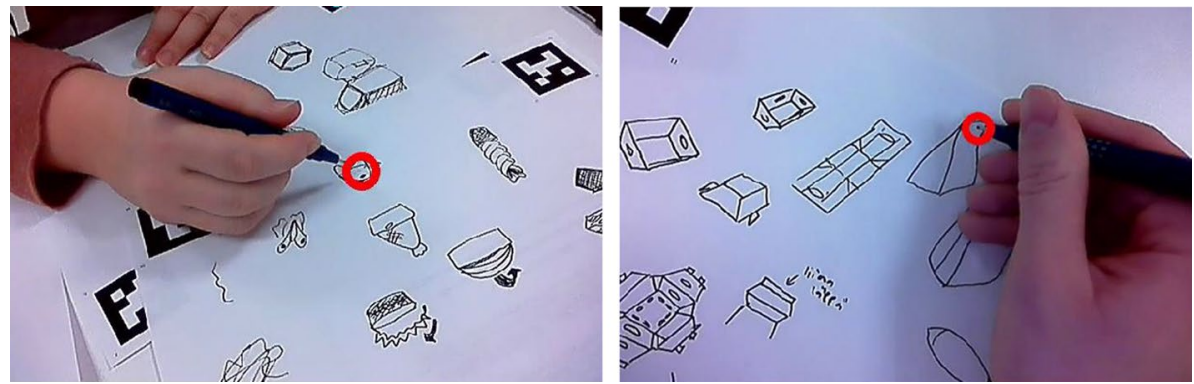

Fig. 4 Two examples of Rita's scene camera data: gaze-overlaid video. The red dot shows the location of Rita's eye gaze. On the left, Rita looks at how Bea points at a sketch on Lisa's sketch sheet. On the right, Rita is looking at her own sketch, which she is finalising

After preparations and a warm-up task, the students were given a design brief that challenged them to design a sustainable Christmas basket that could replace the traditional gourmet gift basket with a reusable or recyclable eco-friendly version. Three tasks were given: (1) to ideate and document ideas with sticky notes, (2) to develop ideas through sketching, and (3) to build a 3D cardboard mock-up of their selected solution. The students worked without time limitations and were free to self-organise their work (at the level of collaboration and their individual work), but they were asked to create a shared solution. After a break, a group interview, stimulated by the gaze-overlaid video data, was started. The objective was to capture students' fresh memories - as accurate as possible - regarding their creative activity during two selected periods with no visible or audible design activity. The first chosen period had occurred during ideation with sticky notes and the second during ideation through sketching. The underlying question was: Which (if any) of the various sketches gazed during the silent periods had provided inspiration for the sketches and ideas that were explicated or sketched immediately after the break. However, the interview did not proceed to the second period, as the interview was interrupted after 25 min when it became clear that the students were too tired to concentrate and to provide this kind of extremely detailed and precise information. The interview data was not used for this study, as the information were on too general level.

This study only used the data from the conceptual design via sketching, which lasted $35 \mathrm{~min}$. This data set comprised students' sketch sheets (a total of 100 sketches), video data from the stationary video cameras (in total, $2 \times 35 \mathrm{~min}$ ) and the gaze-location videos of each student (a total of $3 \times 35 \mathrm{~min}$ and 12,000 fixations). The gaze-overlaid video comprised a video captured by a scene camera (mounted in the middle of the spectacle frame on top of the nose piece) and a gaze-location position that was imposed on top of the scene camera view (see examples in Fig. 4). The overall video recordings were transcribed with f4transkript software. All sketches were labelled with ID numbers.

\section{Integrating verbal, sketch and gaze data into linkography}

In the first phase, the strategy was to create linkographs based on verbal and sketch data and, in the second phase, to utilise the gaze data. The gaze data fulfilled three functions. First, it was used to verify which sketches were being gazed at; second, the timing of 
Phase 1: Creating linkographs based on verbal and sketch data

\begin{tabular}{|c|c|}
\hline Task \& Rules & Outcome \\
\hline $\begin{array}{l}\text { Preparations: } \\
\text { Verbalisations and sketching transcribed from videos. }\end{array}$ & $\begin{array}{l}\text { Transcript. } \\
\text { Sketches labelled with ID numbes, chronological order. }\end{array}$ \\
\hline $\begin{array}{l}\text { Step 1: Verbal moves. } \\
\text { - A conversational turn = a design move. } \\
\text { - Loff-topic remarks }=>\text { remove (cf. Goldschmidt, 2014). }\end{array}$ & $\begin{array}{l}\text { Definition: A move = suggestion, modification, request to } \\
\text { elaborate or clarify, question. } \\
\text { List of verbal design moves, in which a design move } \\
\text { transforms the situation. }\end{array}$ \\
\hline $\begin{array}{l}\text { Step 2: Sketch moves. } \\
\text { - A sketch = a design move. } \\
\text { sketch move with verbal move. }\end{array}$ & $\begin{array}{l}\text { List of verbal and sketch moves, in chronological order. } \\
\text { Note: Moves listed according to start time, if several } \\
\text { participants sketched simultaneously or while one was } \\
\text { speaking. }\end{array}$ \\
\hline $\begin{array}{l}\text { Step 3: Identifying links between moves. } \\
\text { - Moves clearly connected with each other }=>\text { link. } \\
\text { semantomatic" linking of verbal moves based on mere } \\
\text { considered (cf. Bilda et al., 2006). } \\
\text { - Sketch-to-sketch links based on shape analogy, combination } \\
\text { or transformation (cf. Gero, 2000). } \\
\text { - Vague connection => no link. }\end{array}$ & $\begin{array}{l}\text { List of moves complemented with link info } \\
\text { E.g. } \\
\text { Move } 9 \text { provides a modification of an idea presented in } \\
\text { move } 8 \\
\Rightarrow \text { Moves } 8 \text { and } 9 \text { linked. } \\
\text { Move } 40 \text { (sketch) combines ideas presented in move } 21 \\
\text { and shapes in move } 23 \text { (sketch) } \\
\Rightarrow>\text { Move } 40 \text { linked to } 21 \text { and } 23 \text {. }\end{array}$ \\
\hline \multicolumn{2}{|l|}{ Phase 2: Adding gaze data } \\
\hline Task \& Rules & Outcome \\
\hline $\begin{array}{l}\text { Preparations: } \\
\text { - Fixations and fixation targets (i.e. sketches) identified from } \\
\text { gaze videos. } \\
\text { A period starting when a sketch is fixated on and ending } \\
\text { when something else (even blank) is fixated on = a visit. } \\
\text { A period starting when a sketch is fixated on and ending } \\
\text { when another sketch, person or object is fixated on (but can } \\
\text { include fixations on blank area) = a gaze move. }\end{array}$ & $\begin{array}{l}\text { Gaze data reduced to timestamped visits and gaze moves. } \\
\text { List of gaze moves per participant. } \\
\text { Note! Two measures (visit and gaze move) are required for } \\
\text { purposes of triangulation. While visit (dwell) is an } \\
\text { established eye-tracking measure, a gaze move is an } \\
\text { aggregate designed for extended linkography. }\end{array}$ \\
\hline $\begin{array}{l}\text { Step 4: Integrating gaze moves to linkographs. } \\
\text { Gaze moves added to step } 3 \text { linkographs. } \\
\text { vaze move in immediate temporal vicinity of a related } \\
\text { Several gaze moves to a particular sketch }=>\text { keep the first } \\
\text { and remove the others. } \\
\text { No gaze moves to another participant's sketch } X=>\text { sketch } \\
\text { move } X \text { removed from linkograph. }\end{array}$ & $\begin{array}{l}\text { One linkograph per participant. } \\
\text { Linkographs with verbal and sketch moves in chronological } \\
\text { order: One's own sketches according to timing of sketching, } \\
\text { but others' sketches according to timing of first gaze move. } \\
\text { Note: Gaze moves to one's own sketches omitted from } \\
\text { linkographs to preserve linkograph readability. }\end{array}$ \\
\hline $\begin{array}{l}\text { Step 5: Confirming the link system created in step } 3 \text {. Move by } \\
\text { move, check each link. } \\
\text { - For links apparent from verbal or sketch moves, no changes } \\
\text { due to gaze data. } \\
\text { For uncertain or vague links, check the original gaze move } \\
\text { and visit data to determine whether a link exists or not. }\end{array}$ & $\begin{array}{l}\text { One linkograph per participant. } \\
\text { Link system confirmed with gaze data. }\end{array}$ \\
\hline
\end{tabular}

Fig. 5 Phases, steps and rules for compiling multimodal linkographs from verbal, sketch and gaze data

gazing in relation to other linkography moves was determined; and third, it was used to confirm the link system created in phase one. To illustrate individual differences in attending (and benefiting from) 'shared' design activity, gaze data was utilised to create three linkographs - one for each participant. The phases, tasks and rules are elaborated in Fig. 5.

\section{Strict rules and data reduction}

The first phase utilised the same rules as traditional linkographs, with the addition of sketch moves. The rules for the second phase were developed to balance the readability of linkographs with data reduction. Noticing the links between consecutive design moves was usually straightforward, as the conversation often proceeded episodically from one topic to another. However, vaguely presented or extremely fragmented proposals and self-imposed 
constraints caused difficulties. The main rule was that no links were established for vague connections. For instance, a gaze move did not result in a link if no manifest features associated with the fixated sketch were identified in subsequent verbalisations or sketches. A cautious approach was taken to avoid unwarranted claims based on gaze data (cf. Amati \& Brennan, 2018).

Gaze moves that represented re-gazing at sketches later in the course of the designing created a challenge for the linkograph structure. Keeping these gaze moves would have preserved the temporal sequence of speaking, sketching and sketch gazing, but resulted in linkographs with over 300 moves. Goldschmidt (2014) considers a linkograph with 100 moves to be long. Moreover, having 'replicated' moves (a sketch implicated by more than one move) would have compromised the value of linkographs as a powerful tool that renders designers' thinking structure down into a visually expressive format. Rather than just looking at the linkograph, the links to 'replicated' moves would have to be added together to judge the criticality of those sketches. Therefore, the principle that was adopted was that the first gaze move to a sketch could represent all gaze moves to that particular sketch, and the moves due to re-gazing were deleted from the final linkographs.

\section{Confirming the link system: Cautiousness with gaze data}

A linkograph is a practical tool only if it remains readable. Linking requires common sense and good acquaintance with the discipline and the design activity in question, as links should reflect designers' cognitive processing and the structure of their thinking (Goldschmidt, 2014). Figure 6 showcases how gaze data was utilised to confirm interpretations based on verbal and sketch data. Prior to examining this example, we recommend that you study the participants' sketch sheets in Appendix 2. In this study, many sketches embody rather similar shapes, and many features (such as the shape of the bottom, and the shape and location of handles) were studied as different combinations.

The resulting linkographs comprised 100-120 moves. Linkograph visualisations were produced by the LINKODER tool (Pourmohamadi \& Gero, 2011). Moreover, bar charts

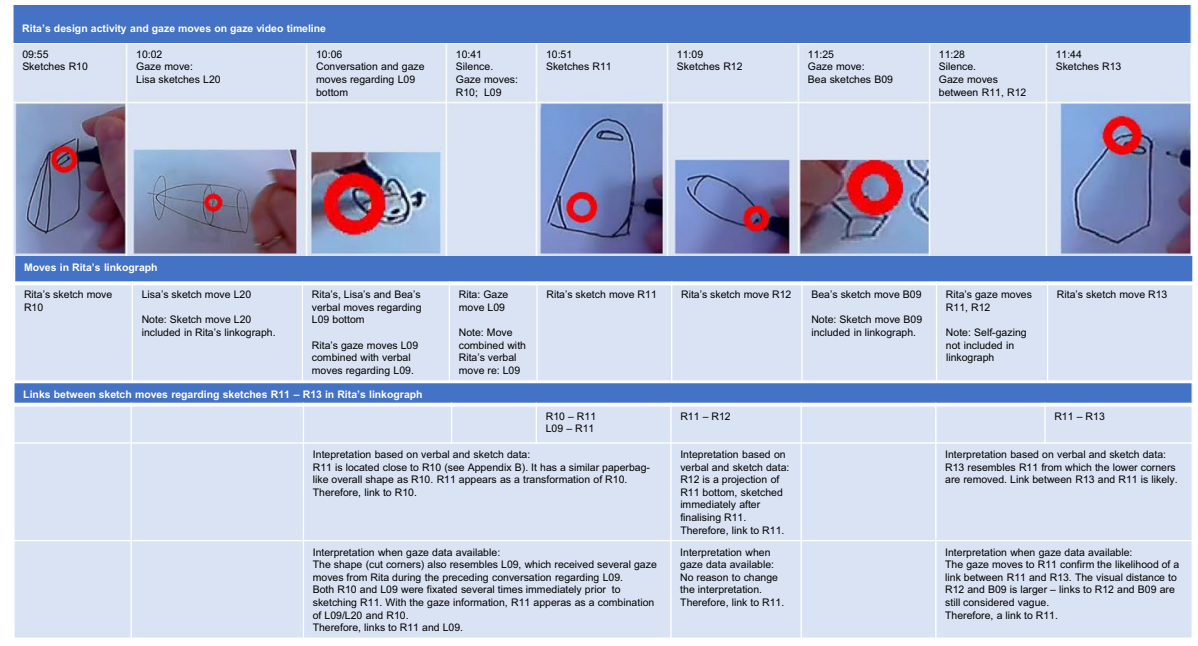

Fig. 6 An example of utilising gaze data to confirm links between sketch moves: Rita's sketches R11-R13 
depicting the frequencies and durations of each participant's visits per each sketch (available in Results, Figs. 9, 11, 12 and 14, 15) were created for triangulation.

The above-described meticulous processing revealed the unfolding of three qualitatively different collaborative design activities that produced two major solution candidates, which were further analysed. The development processes of these solution candidates - one by Lisa and one by Rita - were intertwined through some shared design moves, but collaboration had different roles in these creative processes. To understand the relationship between students' designing and collaboration, a quantitative measure of collaborative design was developed: the Collaboration Index (CoI).

\section{Extended linkography: collaboration Indexes}

The CoI measures the interconnectedness of collaborating designers' design activity - the proportion of the links to other participants' design moves. The maximum value of the index is one, and the closer to one the index score is, the higher the level of connectedness and the level of collaboration are. The $\mathrm{CoI}$ is calculated at the elemental level of an individual design move and can be aggregated to the level of (any chosen) linkography segment or participant.

Moreover, as links to a particular design move can be interpreted as back- and forelinks (Goldschmidt, 2014), two kinds of CoIs can be calculated. Forward-oriented forelinks indicate a move feeding or influencing succeeding moves. These links act as a basis for the Divergent Collaboration Index (DiCoI). Backward-oriented backlinks indicate a move being built on and synthesising prior moves; these links act as a basis for the Convergent Collaboration Index (CoCoI). The calculations for the DiCoI and CoCoI are illustrated in Fig. 7.

The higher the CoI value, the larger the collaborative influence for that particular move, as the higher values require the number of self-links $\left(\mathrm{N}_{\mathrm{R}}\right.$ in the example in Fig. 7) to be smaller than the number of other-links $\left(N_{L}+N_{B} N_{R}\right.$ in the example in Fig. 7). Reaching the maximum index

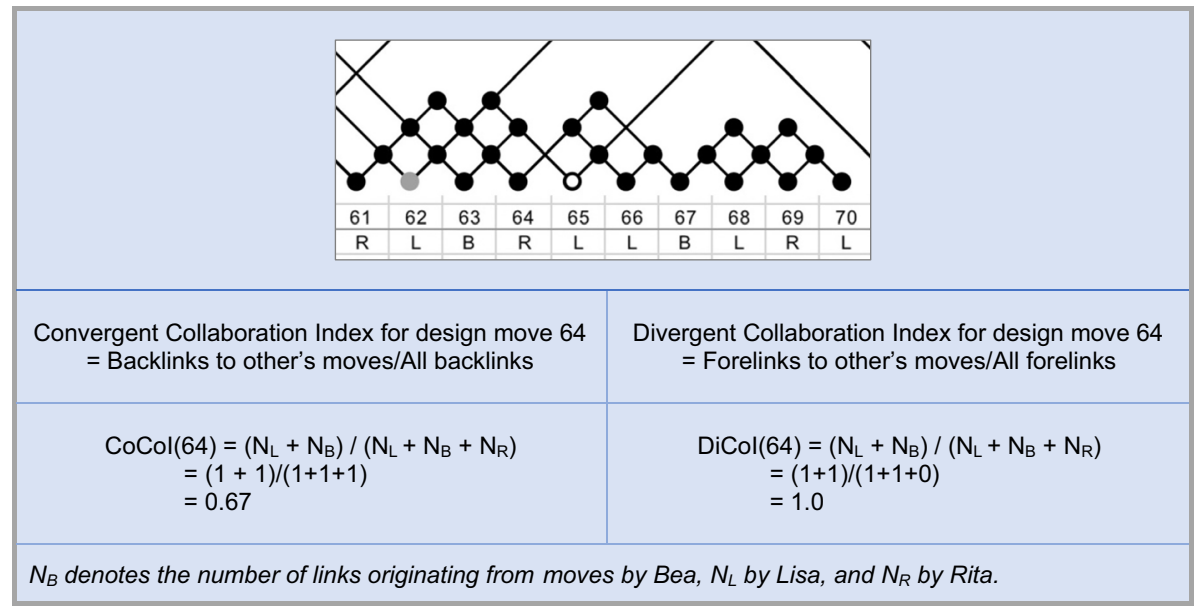

Fig. 7 Calculation of the Convergent and Divergent Collaboration Indexes for move 64 
value (one) is possible only when the number of self-links is zero. The realisation of this collaborative influence requires a two-way engagement from designers: while productive moves have the potential to facilitate and feed others' thinking, the realisation of this potential requires the designers to be attentive to each other's moves. A CoI that remains close to zero suggests that the designers do not benefit from group work but work more or less independently within the group.

$\mathrm{CoI}=1$ All links are to other participants' moves.

$\mathrm{CoI}=0$ All links are to one's own moves.

Once the CoIs per design move are calculated, they can be aggregated to the level of a selected process segment. The aggregated CoI (either the DiCoI or CoCoI) is calculated as a sum of all CoIs (DiCoIs or CoCoIs) within the selected section divided by the total number of design moves within the selected section.

\section{Results}

The design challenge asked the students to deliver a 3D mock-up of their shared solution. They developed several proposals ${ }^{4}$ from which they pointed to two with the intention of building a mock-up of each and then choosing one as their final solution. The two chosen proposals were Lisa's bottle-bottom basket (resembling the bottom of a plastic Coca-Cola bottle) and Rita's foldable one-piece basket (Fig. 8). These two were not their latest proposals, but several others were developed after these two first emerged. However, when prompted to choose, without further ado, the students noted these two as the most prominent. Lisa's, Rita's and Bea's sketch sheets are available in Appendix 2.

The processes through which the two selected proposals emerged represent different types of collaboration that will be described in what follows, first by giving a short overview of the designing and then through linkographs and CoIs. For the linkographs, gaze data was utilised to identify sketches that were given visual attention and links between those sketches. For triangulation purposes, the visual attention paid to sketches was also scrutinised from a more 'traditional' eye-tracking perspective as volumes of visual attention per sketch in visit bar charts. The total number of moves per linkograph was 108-110, the average volume of links per move was 2 and 20 per cent of moves had no back- or forelinks. For CMs, the threshold value was set to 4 (cf. Goldschmidt, 2014). The numbers in parentheses (e.g. 36-39) refer to linkograph move numbers.
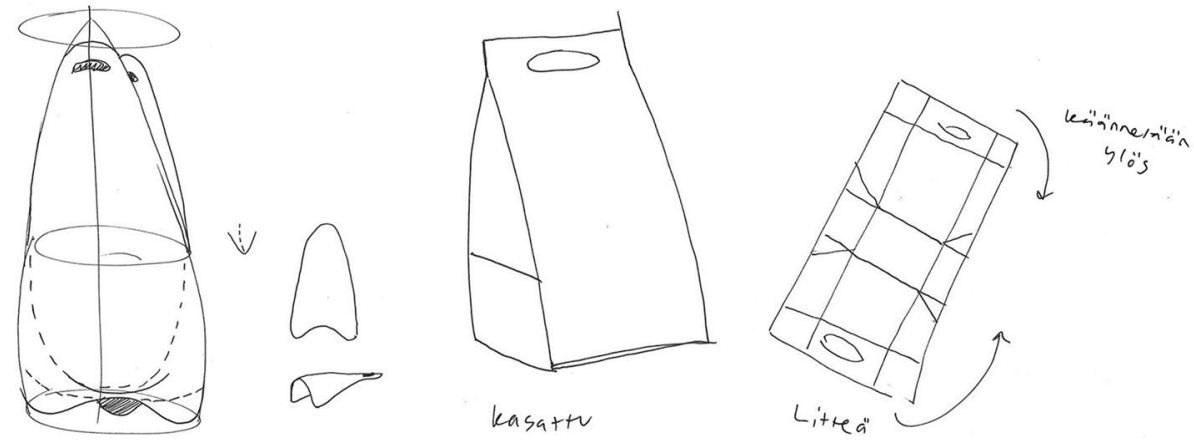

Fig. 8 Chosen proposals: on the left, Lisa's bottle-bottom, and on the right, Rita's foldable one-piece basket

${ }^{4}$ Each sketch is considered to constitute a proposal for a Christmas basket. 


\section{Overview of the designing}

In general, Lisa's designing could be characterised as a versatile and generative exploration, while Rita's designing was focused and foregrounded manufacturing and logistical issues. Both Lisa and Rita sketched actively, but Bea quickly fixated on a couple of shapes and stopped sketching. Bea favoured verbal scenarios and actively supported the development of others' ideas by asking questions and pushing for further details.

While ideating with sticky notes (Task 1), the students actively shared and commented on each other's ideas, producing 37 sketches and 20 written notes. They had treated some topics in detail, often through mini-scenarios describing customer behaviour: effects of sustainability and foldability, and carrying the basket. However, when the students started sketching the proposals (Task 2), their shared process turned into three individual ones that first proceeded rather independently, then merged repeatedly into interactive episodes. All these different configurations were implicitly self-organised by the students - no negotiations were conducted on how to organise the work. Lisa's proposal was developed via one of the interactive episodes, while Rita's proposal mainly emerged silently, paralleling interactive episodes. Episodes refer to distinctive segments, such as developing a particular feature or probing a particular requirement. In linkographs, episodes are often separated by a discontinuity in the link chain (cf. Figures 10 or 13 in which the episodes have been named).

\section{Getting started: co-ordinated sketching}

Lisa, Rita and Bea started with implicitly self-organised co-ordinated sketching. They started and ended their sketching simultaneously without any verbalised agreement and focused on developing their own themes. This six-minute-long coordination was productive - a total of 25 sketches were produced, with only two brief questions interrupting their sketching. It was as if they had silently agreed: 'Let's quickly sketch as many independent proposals as we can, and then stop to evaluate.'

Rita's co-ordinated sketching (Fig. 9) entailed only one episode: she sketched versions of a rectangular, box-like basket bottom and studied folding this kind of one-piece basket.

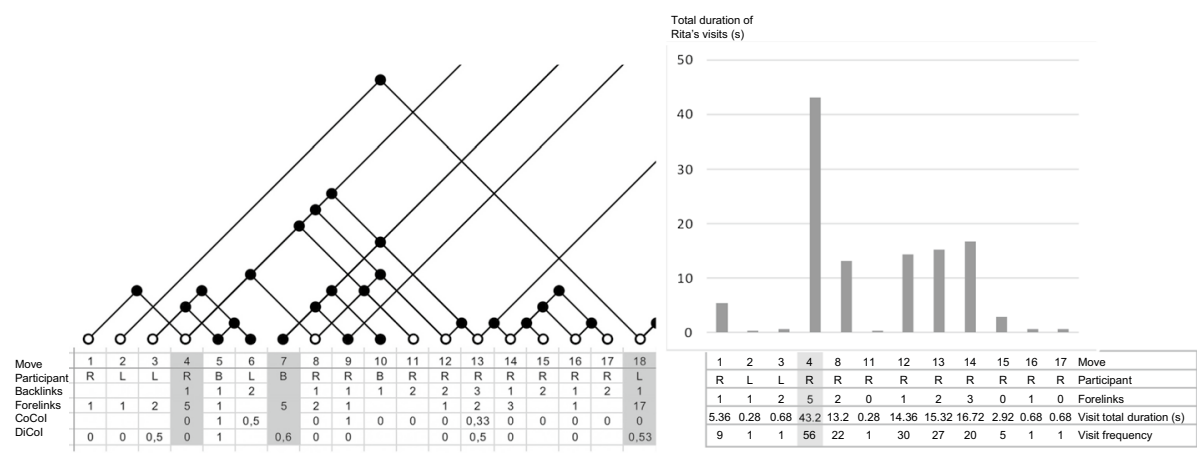

Fig. 9 Rita's independent sketching: sketch moves with links to her own moves, with some fragmented verbal interaction non-related to her own sketching. On the left, is the linkograph, and on the right, a visit chart with the total duration(s) and frequency for Rita's visits per particular sketch (to make comparison easier, sketches are referred to with their move number). Notations: CMs are highlighted in grey, whereas the move colour represents the move type (black: verbal, white: sketch, grey: verbal and sketch) 
The links are mainly between Rita's own sketch moves, and all but one of the aggregated CoI values $(<0.33$ in Table 1$)$ indicate very low interconnectedness. The visit measures concur with the centrality of move 4 and the scarce visual attention Rita directed to Lisa's sketches during this episode.

\section{Lisa: concerted collaborative sketching}

Lisa's proposal (Figs. 10, 11, 12: 18-70) proceeded concertedly as consecutive episodes. In general, the process was shared and iterative: feature by feature, all the exploration was accumulated in Lisa's sketches and in her proposal in Fig. 8. Lisa produced all the related original shapes and structures, and most of the sketched iterations, while everyone actively participated in verbal evaluation and in refining the ideas presented in her sketches.

The link volumes per move are typically low, but a level of connectedness is maintained, with the exception of a few isolated moves $(47,50)$. The most influential moves are sketch moves with the highest visit frequency $(18,40)$, and half of the CMs include sketching. All but one of the CMs reached a CoI value of 0.5 or higher, which indicates interconnected activity. In Table 1, most of the aggregated CoCoIs are higher than the DiCoIs, which indicates that synthesising was more common than feeding others. However, the sketch moves have aggregated CoI values lower than 0.25. According to visit measures (Figs. 11 and 12), Lisa paid very little visual attention to others' sketches, but also many of her own received only a few visits. All but one sketch move in the linkograph was Lisa's. All this indicates that even though structures and features were actively discussed, Lisa's solution was heavily built on her initial sketches (L09 and L10), and that Lisa integrated the features into forms without much visual influence from others.

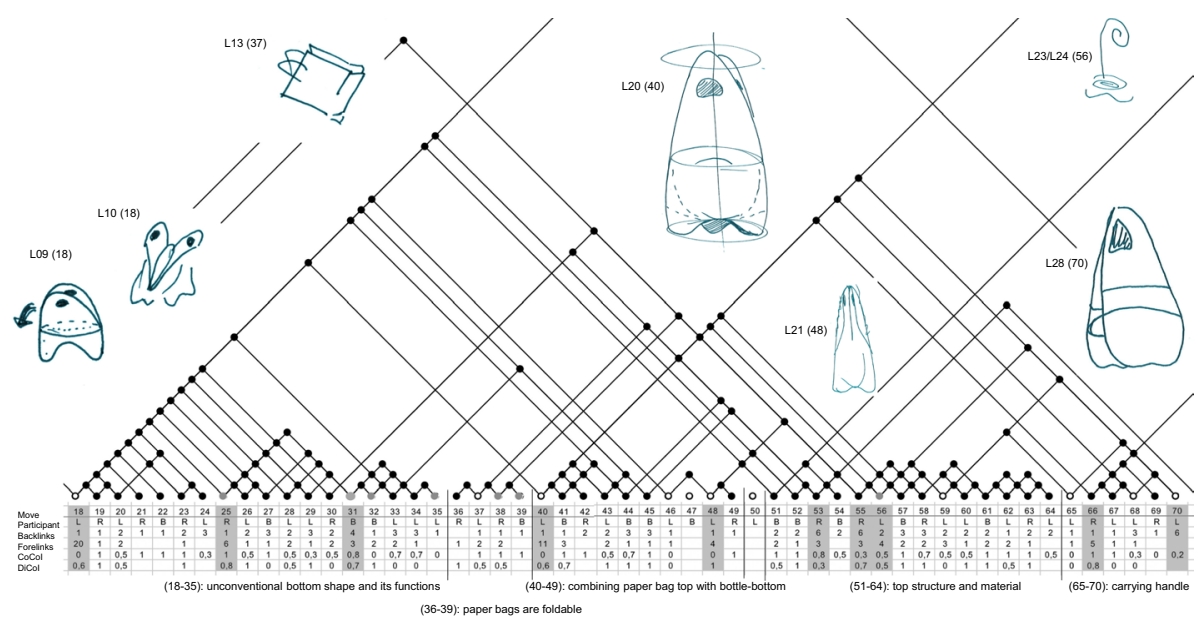

Fig. 10 Development of Lisa's bottle-bottom proposal: Linkograph moves (18-70) and Lisa's central sketches L09, L10, L13, L20 and L28. Notations: CMs are highlighted in grey, the move colour represents the move type (black: verbal, white: sketch, grey: verbal and sketch) and episodes are separated by II marks 


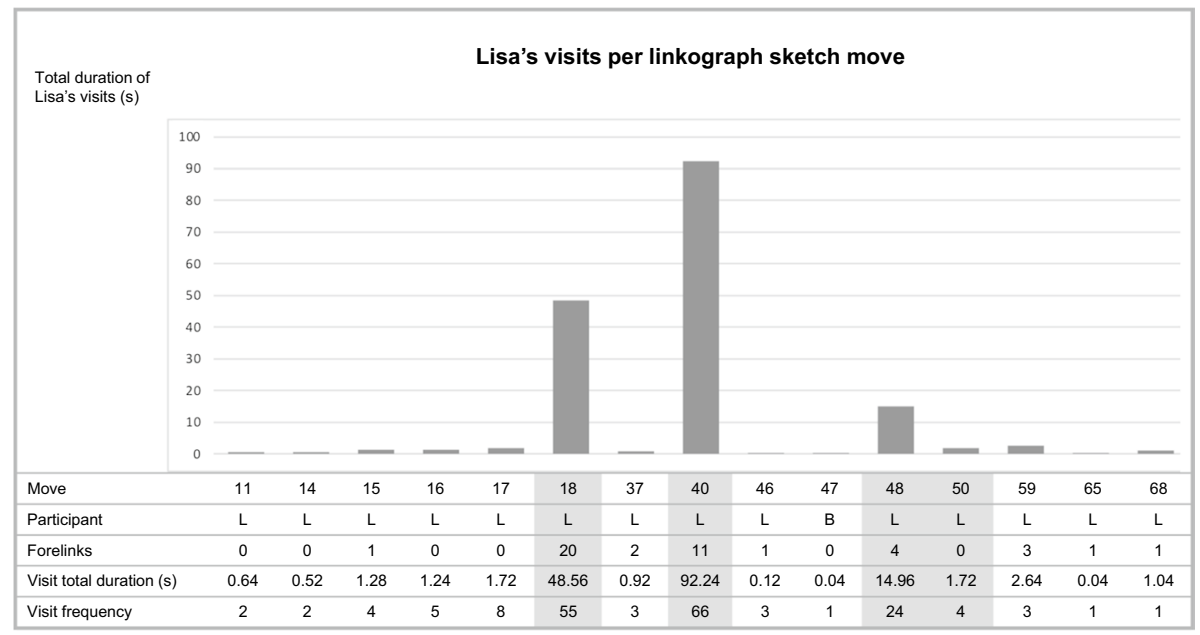

Fig. 11 Lisa's visits during the concerted development of her bottle-bottom proposal (18-70), per linkograph sketch move (marked with white circle in Fig. 10). CM columns are highlighted in grey. This figure shows that Lisa was the one in charge of sketching during this development and that her visits to Rita's and Bea's sketches were scarce (or non-existent) and had low total durations

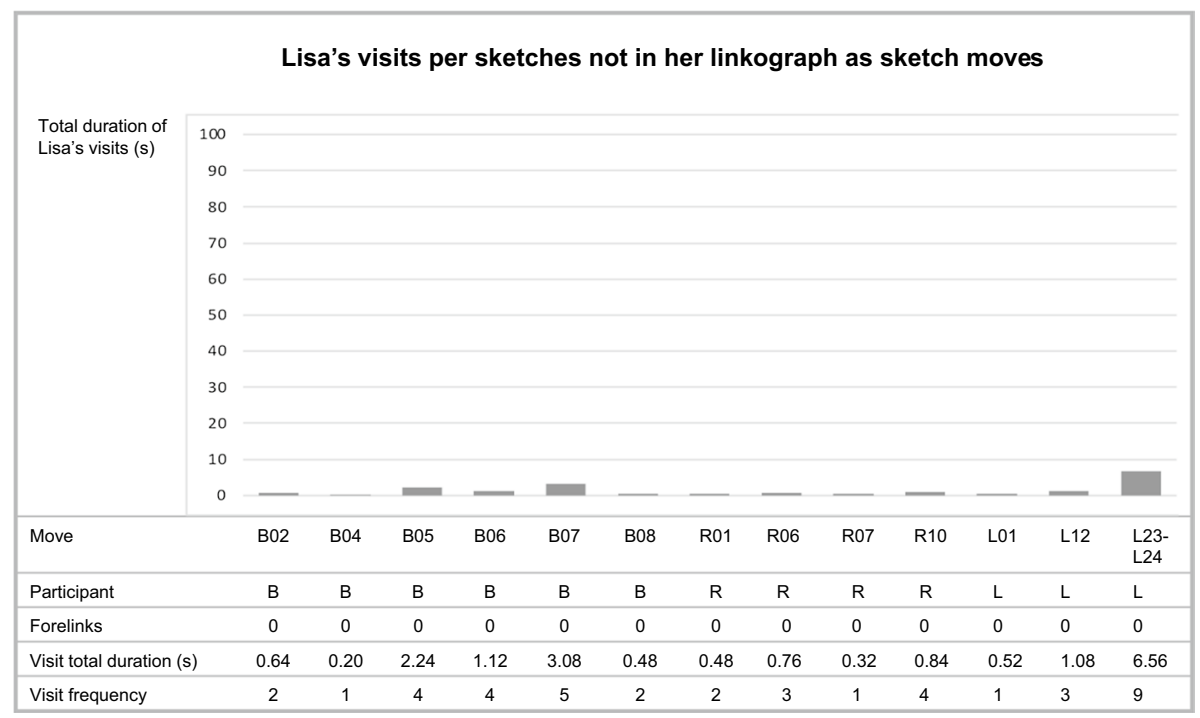

Fig. 12 Lisa's visits per sketches not in her linkograph as separate sketch moves. These visits occurred during conversations related to these sketches, i.e. related gaze moves have been consolidated with verbal design moves (verb + sk, marked with grey circle in Fig. 10). Together with Fig. 11, this figure shows that Lisa's visits to Rita's and Bea's sketches were were scarce (or non-existent) and had low total durations. Unlike in Fig. 10, number of forelinks includes links only from the sketch-part of the consolidated move, whereas forelink frequency in Fig. 10 includes links both from the verbal and the sketch part of the consolidated move. This way the forelink frequency and visit measures of this figure reflect the same phenomena: visual attention and related inspiration. To preserve visual comparability, the vertical axis scale is the same in Figs. 11 and 12. Note: Some sketches very close to each other, both on paper and timewise, have been combined (i.e. L23-L24), as it was impossible to identify the visits reliably. Further, some consolidated (grey) moves contain several sketches, i.e. the number of sketches in this bar chart is not the same as the number of grey moves in Fig. 10 


\section{Rita: sketching asides while participating in concerted collaboration}

Rita participated actively in shared conversation and synthesis $(\mathrm{CoCoI}=0.739)$, but simultaneously developed her own solution through sketching asides centred on a few basic forms (low DiCoI). Asides were based on private and silent explorative sketching, and the developed proposals were introduced to others only after being finalised, or they were not introduced at all.

This development (Figs. 13, 14, 15: 40-110) was started during Lisa's concerted process but continued long after. Rita frequently visited her own early sketches (Fig. 14), and

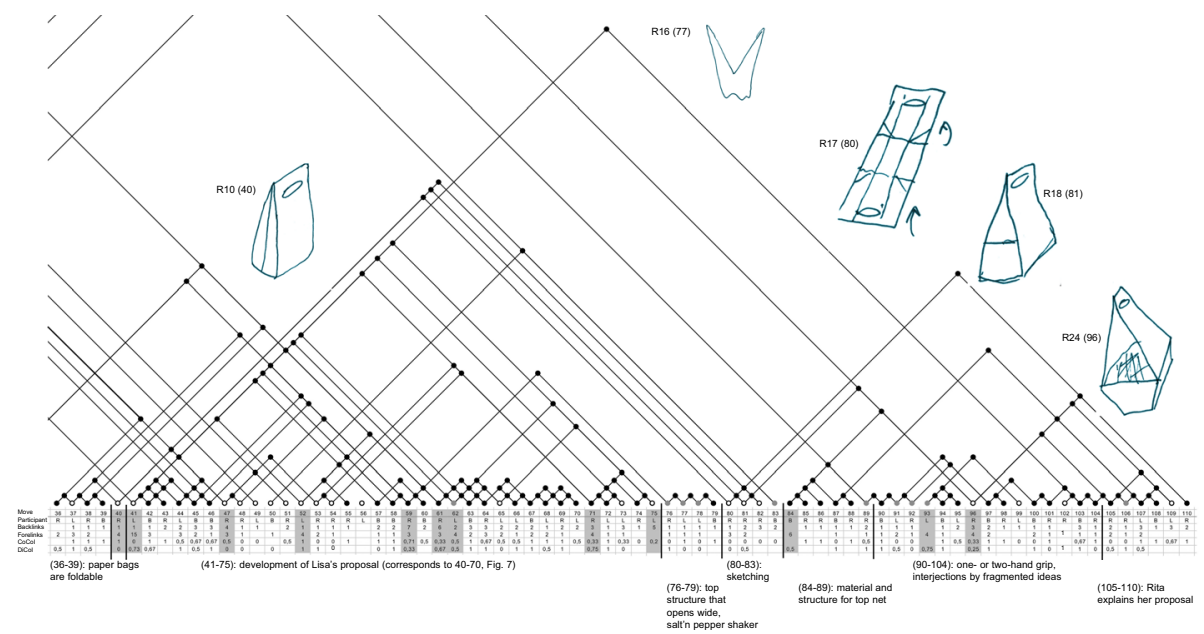

Fig. 13 Development of Rita's foldable proposal: Linkograph moves (36-110) and Rita's central sketches R10, R16, R17, R18 and R24. Notations: CMs are highlighted in grey, the move colour represents the move type (black: verbal, white: sketch, grey: verbal and sketch) and episodes are separated by II marks

the total visit durations were relatively long, yet no obvious links could be identified. Rita brought her proposal to the table only at the very end to explain it rather than to ask for feedback. Others' verbal suggestions had little value for Rita's private process at any point - she had already substantiated several of these suggestions in her sketches before they were collectively discussed. For instance, Rita had already included the location of the grip hole (discussed in 71-74 and substantiated by Lisa in 75) in R10 (40). More than half of the CMs are sketches, yet the most influential ones are Lisa's (L09, L10 and L28).

\section{Summary}

Compared to speech, sketching enables diversification and parallel private processing that is visible as lower aggregated $\mathrm{CoI}$ values. Speech is much more interconnected than sketching is. Forelink-based CMs receive the longest total visit durations, yet for Rita, this was not the whole truth. Rita's private sketching of asides was scattered among the concerted activity. From the video, it can be seen that no pauses in the shared process were caused by these private moves; this sketching of asides did not interfere with shared development. 


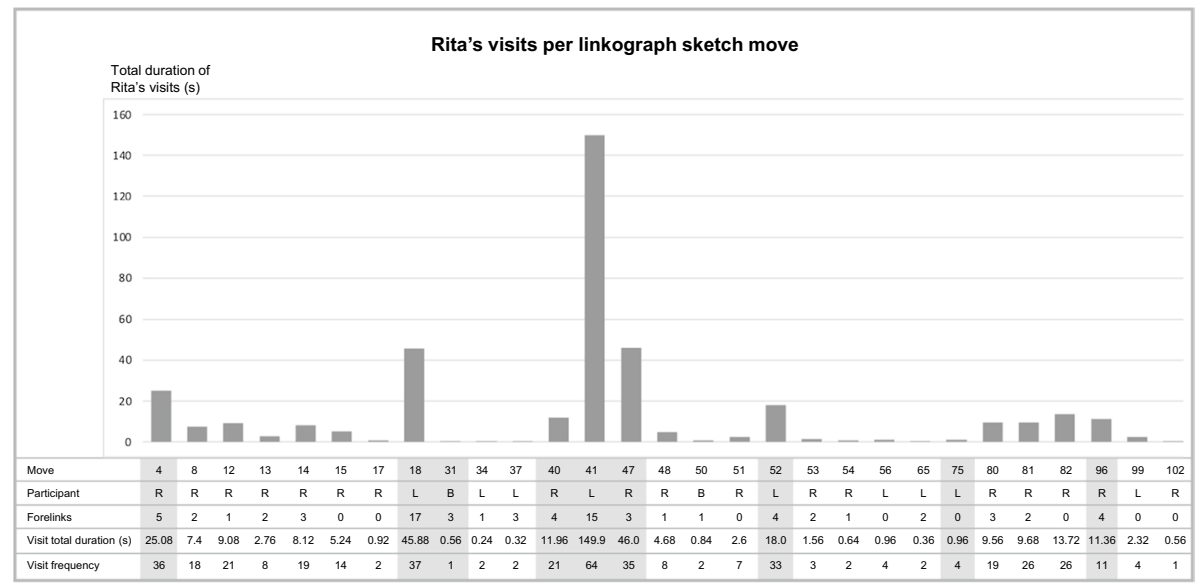

Fig. 14 Rita's visits during the development of her foldable solution (37-110), per linkograph sketch move (marked with white circle in Fig. 13). CM columns are highlighted in grey. Some CMs (e.g. 31) have only a few visits, but in these cases, the criticality is based on backlinks. The figure shows that Rita's visits to Lisa's and Bea's sketches have low total durations, with the exception of sketches belonging to the concerted development of Lisa's bottle-bottom solution (moves 18, 41, 52). Well over half of these visited sketches are Rita's own

To answer the first research question, three different interactive design processes were recognised: (1) co-ordinated: 'silently organised' simultaneous sketching by all three students; (2) concerted: shared verbal exploration that was accumulated in sketches that were collectively evaluated and refined, feature by feature; and 3) aside: private exploration by sketching a candidate that was introduced to others only after being finalised. For co-ordinated sketching, the aggregated $\mathrm{CoI}$ values (all below 0.33 ) are significantly lower than for proposal development. Lisa's proposal development is verbally more convergent, but sketching-wise it was more divergent, while Rita's sketching was more convergent than divergent; Lisa sketched various transformations, but Rita was focused. Further, Rita's aggregated $\mathrm{CoI}$ values for proposal sketching are notably lower than Lisa's, implying a more private activity - sketching asides.

To answer the second research question, the gaze data in the form of visit frequencies and total durations coincides with forelink-based CMs for shared activity and concerted processes. For co-ordinated activity and asides, the visit measures indicate that certain sketches received much visual attention - which suggests that they were important for design activity. However, this suggested importance does not coincide with the volume of links (CMs). The role of these sketches was not to inspire obvious visual derivatives (such as visual analogies, combinations, or lateral or vertical transformations), but to facilitate the process in a way that was not recognised based on the process video and gaze data.

The following section discusses the results and consolidates the role of gaze data in the analysis. 


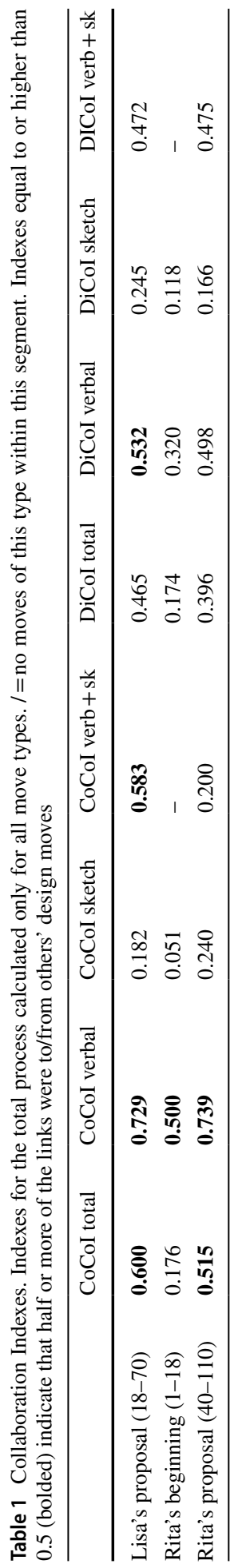




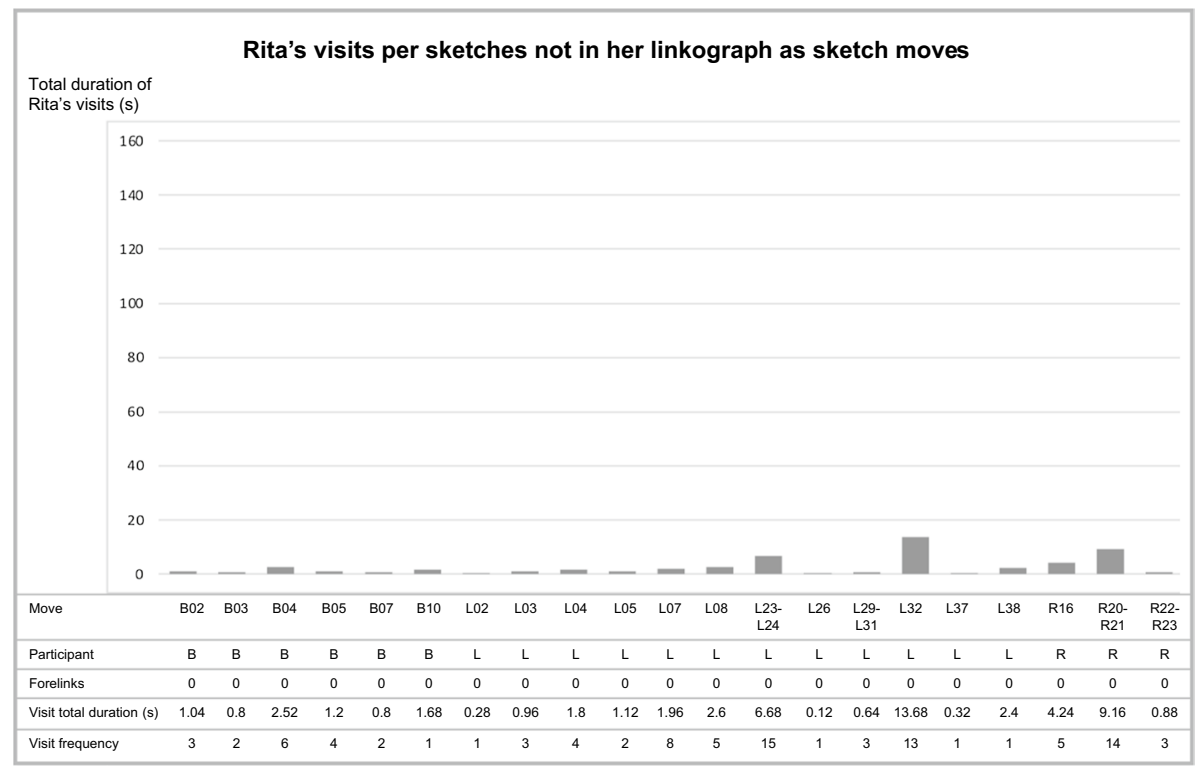

Fig. 15 Rita's visits per sketches not in her linkograph as separate sketch moves. These visits occurred during conversations related to these sketches, i.e. related gaze moves have been consolidated with verbal design moves (verb + sk, marked with grey circle in Fig. 13). Together with Fig. 14, this figure shows how Rita's visual attention was focused mainly on her own sketches, and her visits to these Lisa's and Bea's sketches even shorter than those in Fig. 14. Unlike in Fig. 13, number of forelinks includes links only from the sketch-part of the consolidated move, whereas forelink frequency in Fig. 13 includes links both from the verbal and the sketch part of the consolidated move. This way the forelink frequency and visit measures of this figure reflect the same phenomena: visual attention and related inspiration. To preserve visual comparability, the vertical axis scale is the same in Figs. 14, 15. Note: Some sketches very close to each other, both on paper and timewise, have been combined (i.e. L23-L24), as it was impossible to identify the visits reliably. Further, some consolidated (grey) moves contain several sketches, i.e. the number of sketches in this bar chart is not the same as the number of grey moves in Fig. 13

\section{Discussion}

The developed extended linkograph and Collaboration Indexes enabled the analysis of collaborative design from both targeted perspectives: collaborative interaction and design. Likewise, involving mobile gaze tracking enabled the scrutinisation of collaborative design by balancing verbal design thinking with visual thinking.

In general, the differences in micro-interactional processes - in this study, the level of coordination, sharedness and private asides - led to more or less productive collaboration (Barron, 2003), as the manner and timing of information sharing is different. For successful collaboration, information sharing - making one's thinking publicly accessible - is a necessary condition (Engeström, 1994). In this study, the preliminary understanding was that the development of the two solution candidates was closely intertwined but guided by Lisa's and Rita's different design objectives (the practicality of production, logistics and use for Rita, and inventive and original structures for Lisa). The deeper analysis with the gaze data nudged this interpretation towards a stronger emphasis on individuals' independent creative activity. 


\section{Interactive design processes}

The identified interactive design processes (co-ordinated, concerted, asides) represent different types of interactivity and information sharing, and they complement the patterns of collaborative sketching by Härkki et al. (2018): co-ordinated, collective, disclosed and silenced sketching. In both our studies, co-ordinated sketching entailed an (implied or explicated) agreement to develop individual proposals. However, collective sketching (Härkki et al., 2018) referred to collective exploration by sketching: Each individual sketched some parts, lines and features of the collective team-level solution and decisions were collective, whereas in concerted sketching, the incremental sketching was done by the solution owner (Lisa) after verbal suggestions from others, and the solution ownership remained with Lisa. All the decisions regarding shapes and connections - sketched interpretations of the verbally explicated features - were Lisa's, rather than being collectively made. Sketching asides and disclosed sketching both refer to sketching followed by verbal clarification. In both studies, some sketches remained silenced - not verbally shared or discussed later. According to the gaze data, not only were many sketches silenced by their makers, but they were also bypassed as uninteresting by others, as Rita and Lisa paid relatively little visual attention to sketches that were not discussed.

These behaviours resonate with van der Lugt's (2005) findings from idea-generation meetings that participants do not pay much attention to others' work. When one's own ideas are flowing, this is understandable, and as such, developing one's own ideas does not exclude collaboration. However, independent and shared activity need to be balanced for designing to benefit from collaboration. Schembri et al. (2015) suggested that achieving consensus is facilitated by shared sketches. In this study, Rita's solution was accepted by the others despite her private process - the capacity of the sketch to respond to a given design challenge could be more important than the sharedness. Future research could ask whether silencing a sketch exposes it to bypassing and undermines its value.

\section{Divergent and convergent collaboration}

The CoI values for divergent and convergent collaboration aim to measure the balance between independent and shared activity, as well as changes between idea exploration and synthesis, which is required for designing to proceed towards a solution (cf. a review by Kälviäinen \& Nylander, 2019). Convergence and divergence in design activity are visible in linkographs and can be calculated for segments of interest: per each move, per an episode, per a design session, etc. Goldschmidt (2016) suggested that, in conceptual design, the ratio between divergent and convergent thinking approaches 60:40, and both modes are present throughout the process. Based on the aggregated CoI values of this study, collaborative synthesis was more frequent than collaborative exploration. Most likely, this reflects the data being from a task building on another task: ideation with sticky notes (Task 1). Moreover, Goldschmidt's suggestion was based on data on an individual design activity. Whether the same ratio is applicable for team designing has yet to be validated by further research.

\section{Gaze data and sketching}

The gaze data showed that collaborative sketching activity unfolded differently for different participants - collaboration is not a homogeneous process, as Kvan (2000) noted. Research 
on collaborative problem solving (Hannula, 2016; Hannula \& Williams, 2016) has identified silent periods involving gazing activity that reveal connections between ideas and sketches that, without gaze data, would have remained unnoticed. In this study, most visits to other participants' sketches were related to discussions about those sketches, which is typical when objects in the immediate environment are discussed (Staudte \& Crocker, 2018). Often, one's visual attention also wanders in the near vicinity, which is a likely explanation for visits to sketches that were not included in the personal linkographs (i.e. sketches with no links to other design moves). Typically, longer periods of silent gazing targeted away from one's own work occurred after one's own sketching activity was paused - probably as a sign of running out of ideas or encountering other difficulties (Boyle et al., 1994). In this study, this silent gazing was typically interrupted by another participant asking a question or starting a discussion. These interruptions could be the reason why no signs of these silent gazing periods eliciting sketches building on others' sketches were identified: participants had to choose whether to withdraw to sketch asides or to participate in the discussion - which, in turn, could override the idea that had started to emerge during the silent gazing.

Further, visit measures related to one's own sketches indicate that the linkography links did not capture all types of self-links - a typical problem in observational research (e.g. Wiltschnig et al., 2013). In the absence of a verbal explication (speech, stimulated recall interview data or participant validation), creative design processes, such as emergence and first principles (Gero, 2000), and deep analogies (Casakin \& Goldschmidt, 1999), are difficult to recognise. However, supporting the identification of three out of five creative design processes (Gero, 2000) - visual analogy, combination, transformation - is not insignificant. The gaze data was indispensable in determining personal linkographs and sketch-to-sketch links that represent chains of visual design thinking. Visit measures (high durations and frequencies) that were not illustrated by linkographs indicated that certain sketches were important as anchor points - reference points to visit while thinking - and as possible stimuli for the emergence or use of first principles. Future research could scrutinise the role of these anchors in designers' visual thinking and push the limits of observable design behaviour even further with the additional help of stimulated recall interview data or participant validation.

\section{Conclusions}

Our long-term objective is to support theory-building of collaborative design as an interactive endeavour and to recognise productive interaction that supports design creativity, which has implications for design theory, education and methodology.

\section{Methodological implications}

The developed extended linkograph, especially CoI values, has potential value for research on design education and design expertise, but also on collaborative problem solving in other domains. As such, the method facilitates capturing the variety of interactive design processes involving various $2 \mathrm{D}$ and $3 \mathrm{D}$ design representations. For instance, the success rate of an educational intervention centring on sketches and sketching could be measured by pre- and post-tasks (designing) analysed with the developed method.

From a methodology development viewpoint, two very different focus areas exist: beneficial interaction and the individual designer's silent processing. The first, having top priority on our agenda, includes research questions regarding sharing and attending to ideas 
(in multiple modalities), the depth of exploration and knowledge integration, and recording co-constructed knowledge. The latter involves questions related to self-links, anchor points and silent processing. A deeper analysis of beneficial interaction could involve developing suitable coding schemes, as discussed later. Scrutinising designers' silent processing requires combining observational studies with in-depth interviews or, for instance, neuroscientific methods. Our priorities are based on a belief that for design education, studies on the first focus area could provide important practical results relatively easy to integrate into curricula.

\section{Limitations of this study}

The gaze tracker had its own limitations (detailed below), but the primary sources of limitations were the decisions regarding data reduction, and defining a design move and a link. However, data reduction was necessary to keep the number of moves and links reasonable and the linkographs readable. Further, the length of the study caused the group interview to be prematurely interrupted as it was non-productive. Therefore, this case study is purely based on observational (video) data.

According to Yin (1989), a small sample size is not a barrier to external validity, provided that the study is detailed, and that the data analysis reveals elements of practice that are relevant to the study at hand. This sample of one team produced 100 sketches and over 12,000 fixations over $35 \mathrm{~min}$, which could be considered ample data for a study involving mobile gaze tracking. However, the findings of this case study are not meant to be generalised. Through evaluating the similarity between this case description and their own research design, researchers may relate the findings to their own work, but more importantly, they can assess the suitability of the developed constructs in meeting their own purposes.

The mobile gaze tracker was developed at the Finnish Institute of Occupational Health to provide a cost-effective, open-source device that is more accurate and robust than commercial alternatives (Toivanen et al., 2017). The tracker frame increased data quality but restricted the field of vision, which resulted in a loss of data when fixating on an area that was close to (the edge of the table and) one's body. ${ }^{5}$ This could have resulted in a loss of self-links that were otherwise vague and could have been confirmed based on the gaze data.

The study was of considerable length (for a mobile gaze-tracking study), which resulted in the tracker frames causing some discomfort to participants. Two of the students said that, after a few minutes, they were no longer aware of the glasses. The third student, despite the clear discomfort, did not feel that the trackers affected her design behaviour or visual attention. In the future, soft nose pads should be available.

From all the data-reduction rules (as explicated in Fig. 5), the ones with the most impact on gaze data are elaborated below:

First, only fixations longer than three consecutive video frames $(120 \mathrm{~ms})$ were translated into gaze moves. This is typical for eye-tracking research. As the typical fixation durations in collaborative problem solving vary between 125 and $150 \mathrm{~ms}$ (Hannula, 2016), it is assumed that all relevant gazing was incorporated.

\footnotetext{
${ }^{5}$ This limitation is shared among gaze trackers with a rim below the 'lens' area, and the effect was mitigated by lining the table edge with thick fleece (grey lining in Fig. 2). However, especially in Rita's case, this was not enough.
} 
Second, sketches with no gaze moves were omitted from linkographs. This resulted in the removal of one-third of all sketches. For transparency, visit frequencies and total durations per sketch are available in the visit charts (Figs. 11-12 and Figs. 14-15). It is possible that some sketches that were not fixated on were still seen and processed, as they were near other, fixated sketches. While movement and larger objects can be recognised by peripheral vision, the field of vision with very good acuity is narrow (the foveal area, the central $2^{\circ}$ of vision), and a good level of acuity is only somewhat larger (the parafovea, $5^{\circ}$ on either side of the fixation) (Rayner, 1998). Therefore, it is assumed that all relevant visual processing was included.

Third, gaze moves to participants' own sketches were omitted, as the main research interest lay in the interaction between participants. However, the data was used to confirm links.

The principles for creating design moves are elemental for linkograph reliability, according to Perry and Krippendorff (2013). This study benefitted from the collaborative setting, which provides clear basic boundaries for moves: conversational turn taking, the creation of a sketch, adding new features to a previous sketch and gazing at someone else's sketch that is not currently under discussion are all modality-based 'syntactical' actions and are objectively observable. ${ }^{6}$ Some 'syntactical' verbal moves had to be divided, as keeping obviously separate topics within one move could confuse the link system.

When creating links, the major principle was that for vague connections, no links were established. The links were created by only one researcher, but twice: six months in between the first and the second time. The intra-rater reliability was good: the differences were of minor magnitude (less than $2 \%$ of the total link volume), but more importantly, they were related to moves containing fragmented elaborations (cf. Hatcher et al., 2018) and did not change the set of CMs. The reliability of the links was improved by triangulation of the modalities: verbalisations, gestures and sketches, as well as gaze data. However, we recommend engaging several researchers and a more complicated process (elaborated below), especially when the generalisability of results is attempted, as the reliability of the developed $\mathrm{CoI}$ measures depends on the reliability of moves and links.

\section{Methodological recommendations}

To assess the reliability of a linkograph, Goldschmidt (2014) recommends that three independent judges determine the links, following a discussion solving their disagreements. More sophisticated procedures for determining inter-rater reliability values for design moves and links have been developed by van der Lugt (2005), Blom and Bogaers (2020) and Hatcher et al. (2018). ${ }^{7}$ The most rigorous approach by Hatcher et al. (2018) engaged two independent judges to transcribe videos, segment design moves and create links. Then, all these phases were cross-checked, and agreements and disagreements were recorded in order to calculate Fleiss' kappa.

However, for the developed extended linkographs, rules related to gaze data (Phase 2 in Fig. 5) should also be evaluated. This complicates the process considerably and makes it (even more) laborious. Our suggestion is to utilise two trained but independent judges,

\footnotetext{
${ }^{6}$ Perry and Krippendorff (2013) conclude that parsing design moves from dialogue has advantages over parsing from monological think-aloud protocols, which suggests that collaborative design processes could be easier to parse than individual design processes.

7 Readers interested in all the details of these processes should refer to the original publications.
} 
going through all the steps in Fig. 5 for (at least) 20 per cent of the design challenge length, discussing the disagreements and carefully documenting underlying reasons (Hammer \& Berland, 2014), such as vague rules or segment ambiguousness, which should then be processed to refine the rules. Then, a suitable reliability coefficient for linkograph links should be calculated. For instance, Krippendorff (2011) criticises commonly used reliability coefficients (such as percentages and Cohen's kappa) for neglecting the effects of agreement by chance and recommends utilising Krippendorff's alpha.

Another recommendation concerns end interviews. Lyle (2003) questions the tendency to treat stimulated recall interviews as unproblematic and highlights the different challenges of stimulating reflection (for instance, strategies or decision making) and stimulating recall. In this study, the objective for stimulated recall interview was to reach fresh memories (as accurate and complete as possible) about which sketches (or features of sketches) had during the two selected periods acted as springboards to creative processes (such as emergence or identifying structural, functional or behavioural analogues) that followed immediately after the silent periods. A task requiring deep concentration, stimulating participants with (an unfamiliar representation such as) gaze-overlaid video showing possibly unconscious eye-movements, and asking to explicate possibly unconscious use of analogy or emergence (or other such creative processes) could be a challenge even for an active and rested mind. To further highlight the complicatedness of the task, over 40 sketches had already been produced prior to the first silent period selected for the interview, and $15-35$ of those sketches had been gazed during the period. Analysing one's own exploration at this level of detail is an unusual task. Unsurprisingly, after over two hours of designing, the students were too tired to reach their memories at this fine level of detail. The interview was interrupted. Further postponing this recall task to another day would not have increased the validity of the interview data (cf. Lyle, 2003) but burdened students. Even if it might prove to be overly optimistic, we propose that participants should be interviewed individually immediately after the design tasks. The questions could focus on the discontinuity of sketching: pauses, sketches with markedly differing features, or consecutive sketches not belonging to a set of 'visually obvious' lateral or vertical transformations. A more resource-friendly alternative to increase study validity could be to ask participants to validate the linkography links determined by researchers.

\section{Implications for design theory and education}

Despite growing interest in collaboration and collaborative design in education, both collaboration (Tessier, 2020) and collaborative design (Kiernan et al., 2020; Kvan, 2000) are notions that are used flexibly. Collaboration can refer to a situation (i.e. a possibility) or type of interaction (i.e. actual team performance). Researchers and design educators should be more specific, as this conceptual ambiguousness hinders the development of a theoretical understanding of design collaboration and its benefits, but also the development of effective teaching practices. For instance, in an attempt to capture 'good collaboration', Détienne et al. (2012) developed a rating tool with dozens of questions for external judges, only to notice later (Détienne et al., 2017) that the ratings were dependent on local practices, norms and values. Good collaboration varied from culture to culture. How does this translate into the growing volume of multinational teams and projects?

Frameworks supporting teaching collaborative interaction exist (cf. Kiernan et al., 2020), but their suitability for design education needs to be reviewed, case by case. 
Currently, there is meagre consensus on 'good' collaboration, 'good' design, or the relationship between these two. Therefore, this research suggests that rather than aiming to capture 'good' collaborative design, educators could teach students to design collaboratively in such a way that the benefits of teamwork are maximised (or at least, well capitalised on) in that particular situation, with that particular task, team composition and culture. We suggest supporting this aim by engaging students in evaluating their own collaboration and by analysing students' collaborative designing by methods such as those described in this study. That would provide two perspectives on actualised collaboration and benefit the further development of theory and teaching guidelines.

Maximising the benefits most likely entails a set of strategies. Kälviäinen and Nylander (2019) suggested that design education should provide students with an understanding of various stages and a diversity of design methods, as well as how to combine those pieces to meet the contextual, material, immaterial and organisational design challenges. While the major challenge lies in supporting students to understand their sources of creativity, design educators also need to support students to collaborate creatively (Kälviäinen \& Nylander, 2019). This study revealed that the three students collaborated, designed and sketched differently; they attended to each other's sketches differently. If this result reflects their general performance, they could benefit from slightly different educational support activities.

Tessier's (2020) review on collaborative design emphasised the importance of knowledge exchange that results in learning. A review on team innovation by Thayer et al., (2018, p. 363) emphasised that the value of collaboration is based on 'the ability to draw from diverse perspectives and expertise'. They recognised knowledge integration as the prerequisite for benefiting from this diversity and emphasised that focusing on information sharing and the volume or frequency of communication is not enough. Similarly, Sauder and Jin (2016) suggest that critiquing followed by elaboration or revising inspires more thought processes than merely 'building on'. This is exemplified by the concerted process identified in this study. Further, future research will feature Bea's valuable collaborative role (questioning, pushing others to refine and revise). In the described concerted process, sketches and sketching were repeatedly utilised as repositories and tools for a shared deepening exploration. This resonates with representations understood as recordings of co-constructed knowledge (Cash et al., 2020). In the future, an analysis of the depth of exploration and knowledge integration could be integrated into the extended linkograph. Recognising whether CMs are based on links reflecting deeper or more surface-level processing could be based on an additional coding scheme for moves and links.

In collaboration, participants' activity becomes interrelated, even interdependent (Cash et al., 2020), yet individual agency does not disappear. In the developed extended linkographs, CoI measures signify the integration of knowledge. Links connecting different participants' activities imply sharing and attentiveness to each other's contributions and collaborative knowledge integration, which are prerequisites for beneficial collaboration. In an attempt to clarify the concept of collaboration, this research coins intense collaborative design as active participation and the mutual facilitation of each other's design activity, with the aim of fully harnessing every participant's knowledge and experience for knowledge integration and for creating a satisfying design. 


\section{Appendix 1}

Initial questionnaire.

Background information for Gaze Tracking in Design

Welcome to the pilot study Gaze tracking in Design. With this form we collect background information from the participants

Personal data

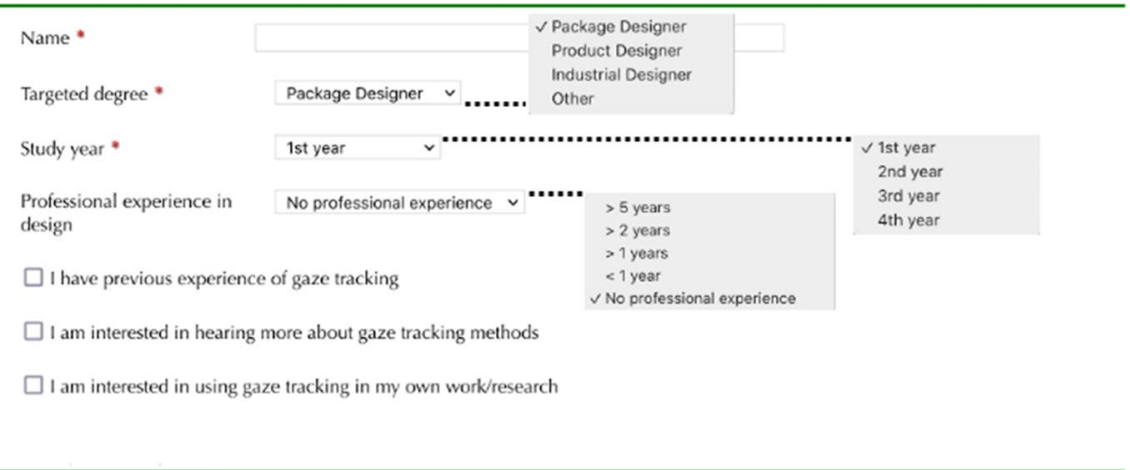

\section{Appendix 2}

Lisa's 1st sketch sheet. 


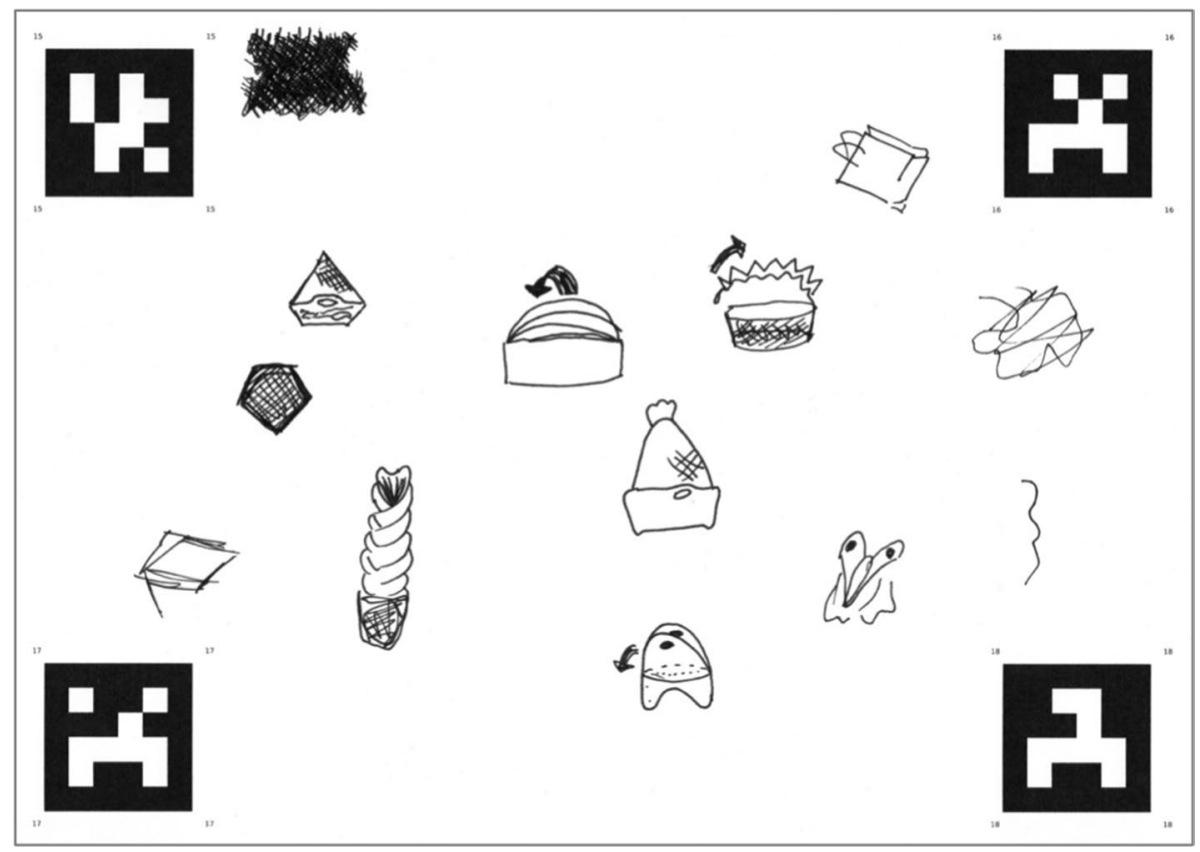

Lisa's 2nd sketch sheet. 


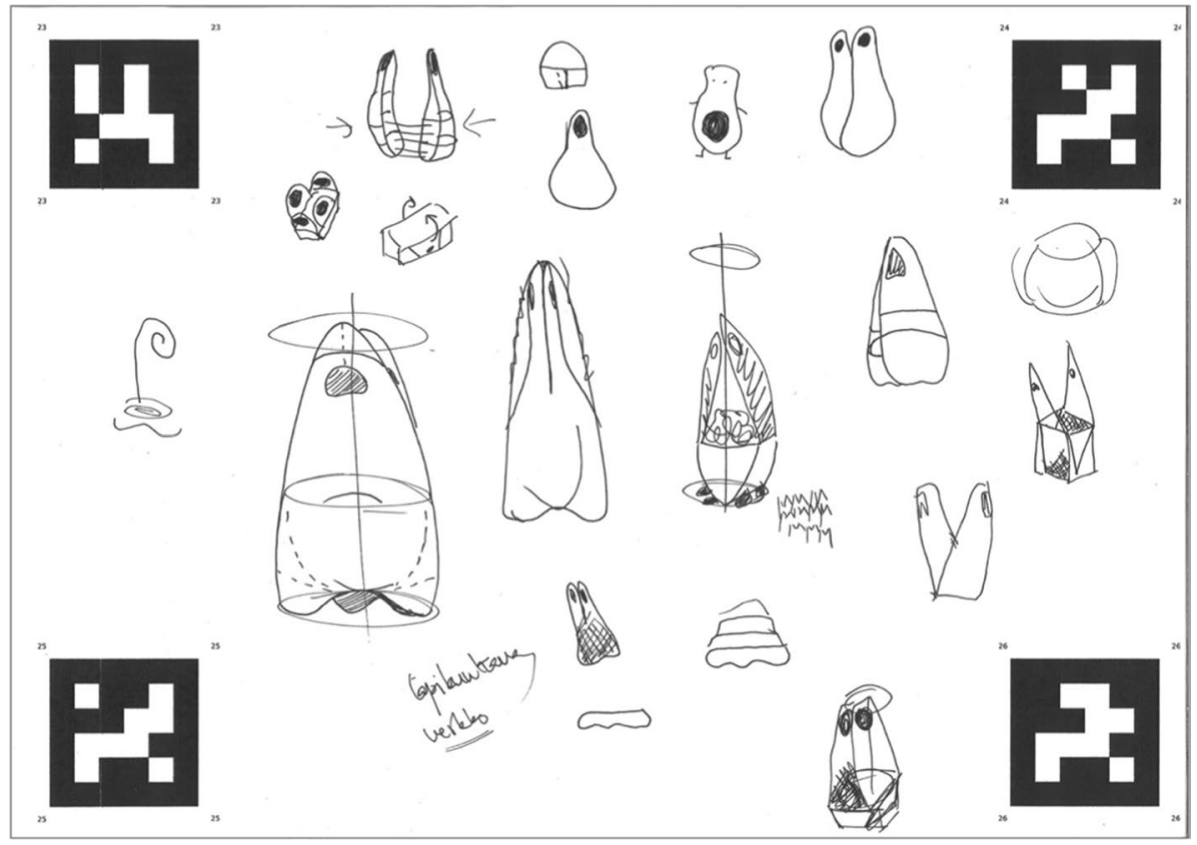

Rita's sketch sheet. 


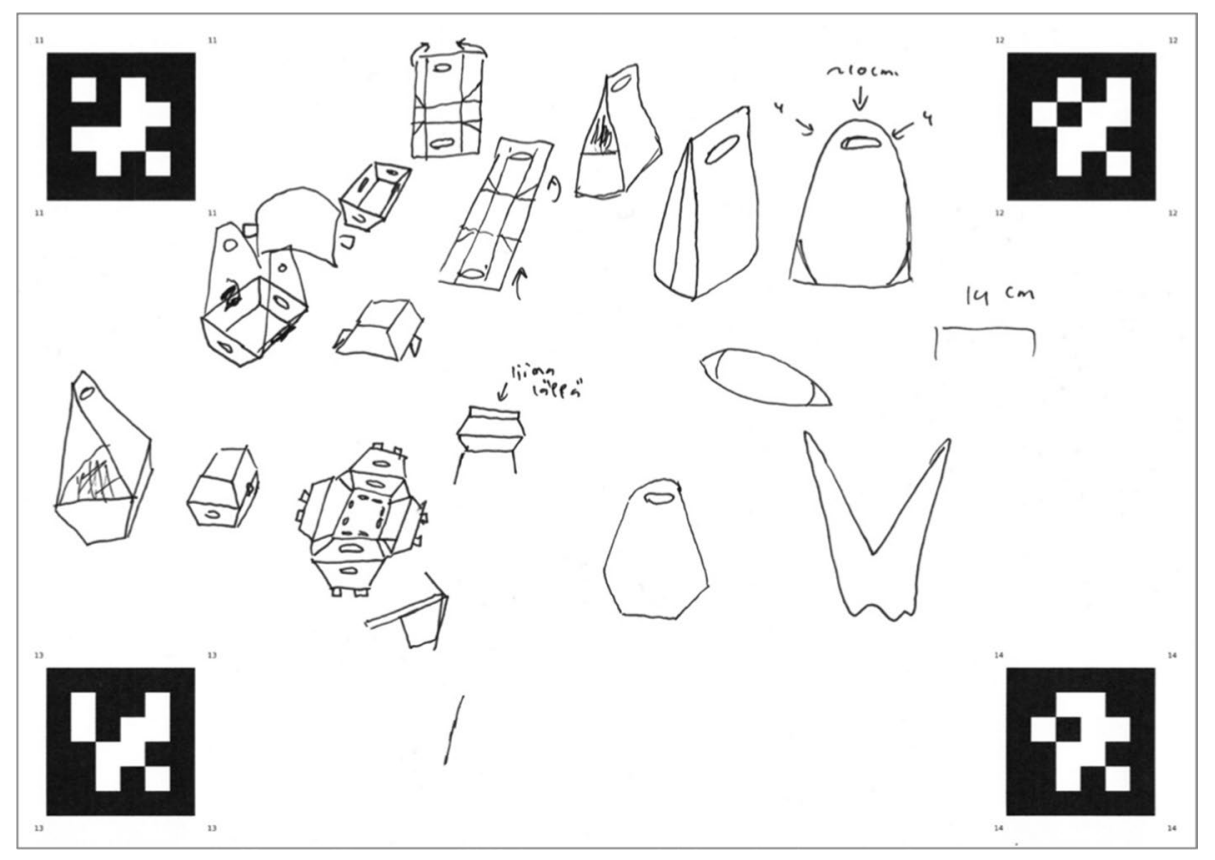

Bea's sketch sheet. 


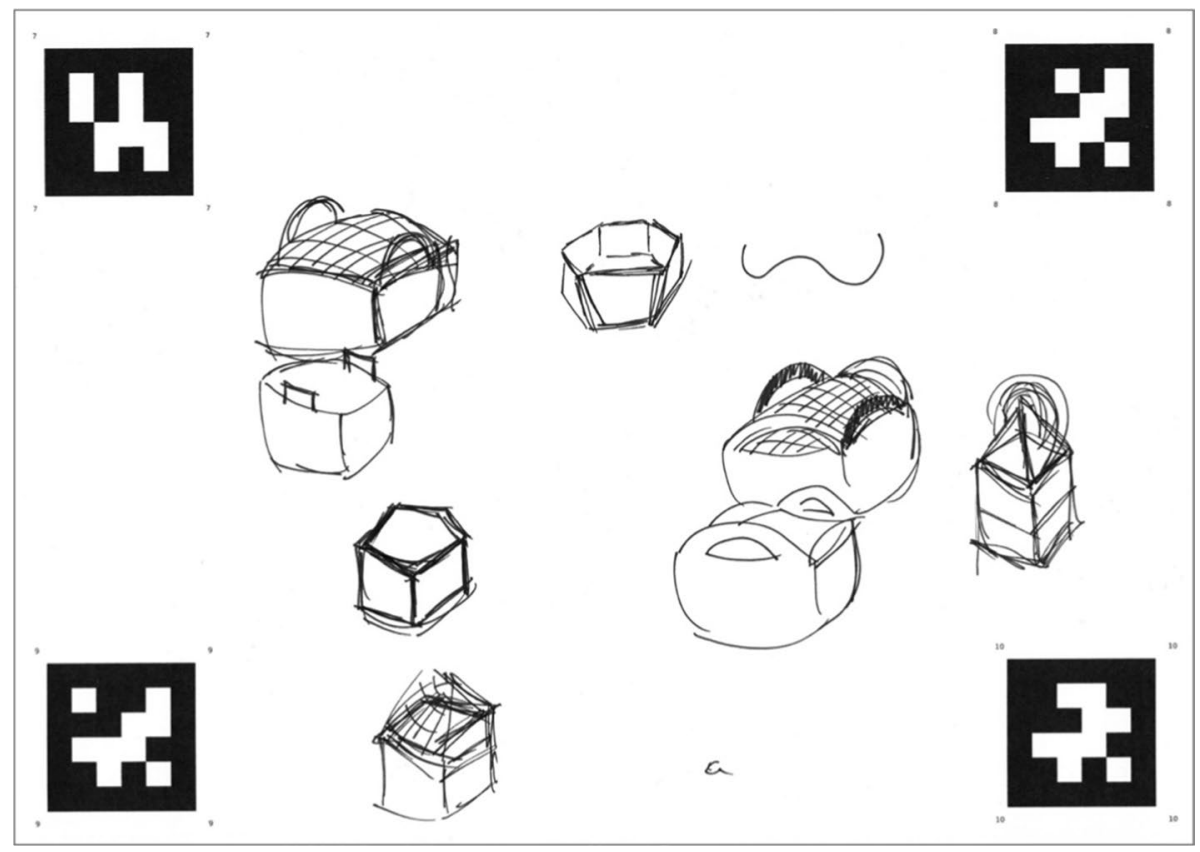

Acknowledgements I would like to thank the participating students, as well as the LAB University of Applied Sciences for providing facilities for the research setting. I am especially thankful for the valuable time and energy of Packaging Design Lecturer Noora Nylander. For providing the gaze-tracking apparatus and expertise, I thank Professor Markku Hannula and Enrique Garcia Moreno-Esteva and the technical wizard Visajaani Salonen from the MathTrack project. This research would not have been possible without their time and expertise. Further, I would like to thank Professor Gabriela Goldschmidt for reading an early version of the manuscript, as well as the two anonymous reviewers and the editor for providing valuable critical questions, recommendations, and encouraging support.

Funding This research has been funded by the Emil Aaltonen Foundation, the Aino-koti säätiö and the Academy of Finland (grant 297856). Open Access funding provided by University of Helsinki including Helsinki University Central Hospital.

Open Access This article is licensed under a Creative Commons Attribution 4.0 International License, which permits use, sharing, adaptation, distribution and reproduction in any medium or format, as long as you give appropriate credit to the original author(s) and the source, provide a link to the Creative Commons licence, and indicate if changes were made. The images or other third party material in this article are included in the article's Creative Commons licence, unless indicated otherwise in a credit line to the material. If material is not included in the article's Creative Commons licence and your intended use is not permitted by statutory regulation or exceeds the permitted use, you will need to obtain permission directly from the copyright holder. To view a copy of this licence, visit http://creativecommons.org/licenses/by/4.0/.

\section{References}

Amati, F., \& Brennan, S. E. (2018). Eye gaze as a cue for recognizing intention and coordinating joint action. In G. Brône \& B. Oben (Eds.), Eye-tracking in interaction (pp. 21-46). John Benjamins.

Barron, B. (2003). When smart groups fail. Journal of the Learning Sciences, 12(3), 307-359. 
Bilda, Z., Gero, J. S., \& Purcell, T. (2006). To sketch or not to sketch? Design Studies, 27, 587-613.

Binkley, M., Erstad, O., Herman, J., Ripley, M., Miller-Ricci, M., \& Rumble, M. (2012). Defining twentyfirst century skills. In E. Care, P. Griffin, \& B. McGaw (Eds.), Assessment and teaching of 21st century skills (pp. 17-66). Springer.

Blom, N., \& Bogaers, A. (2020). Using linkography to investigate students' thinking and information use during a STEM task. International Journal of Technology and Design Education, 30(1), 1-20.

Booth, J. W., Taborda, E. A., Ramani, K., \& Reid, T. (2016). Interventions for teaching sketching skills and reducing inhibition for novice engineering designers. Design Studies, 43, 1-23.

Boyle, E. A., Anderson, A. H., \& Newlands, A. (1994). The effects of visibility on dialogue and performance in a cooperative problem solving task. Language and Speech, 37(1), 1-20.

Brun, J., Le Masson, P., \& Weil, B. (2016). Designing with sketches. Design. Design Science, 2, e13.

Cai, H., Do, E.Y.-L., \& Zimring, C. M. (2010). Extended linkography and distance graph in design evaluation. Design Studies, 31, 146-168.

Casakin, H., \& Goldschmidt, G. (1999). Expertise and the use of visual analogy. Design Studies, 20, $153-175$.

Cash, P., Dekoninck, E., \& Ahmed-Kristensen, S. (2020). Working with the beat. Design Studies, 69, 100943.

Cross, N., \& Clayburn Cross, A. (1995). Observations of teamwork and social processes in design. Design Studies, 16, 143-170.

Darses, F., Détienne, F., Falzon, P. \& Visser, V. (2001). A method for analysing collective design process. INRIA.

Dede, C. (2009). Comparing frameworks for "21st century skills". In J. Bellance \& R. Brands (Eds.), 21st century skills: Rethinking how students learn (pp. 51-76). Bloomington, IN: Solution Tree.

Deininger, M., Daly, S. R., Sienko, K. H., \& Lee, J. C. (2017). Novice designers' use of prototypes in engineering design. Design Studies, 51, 25-65.

Détienne, F., Baker, M., Vanhille, M., \& Mougenot, C. (2017). Cultures of collaboration in engineering design education. International Journal of Design Creativity and Innovation, 5(1-2), 104-128.

Dillenbourg, P. (1999). What do you mean by collaborative learning? In P. Dillenbourg (Ed.), Collaborative learning: cognitive and computational approaches (pp. 1-19). Elsevier.

Dunn, J., Salmela-Aro, K., Talreja, V., Abiko, T., Steinemann, N., Leadbeater, C., ... Bishop, P. (2017). Education 2030-Conceptual learning framework. OECD. Downloaded from https://www.oecd.org/ education/2030project/contact/Conceptual_learning_framework_Conceptual_papers.pdf

Eckert, C., Blackwell, A., Stacey, M., Earl, C., \& Church, L. (2012). Sketching across design domains. Artificial Intelligence for Engineering Design, 26(3), 245-266.

Engeström, Y. (1994). Teachers as collaborative thinkers. In E. Carlgren, G. Handal, \& S. Vaage (Eds.), Teachers' minds and actions (pp. 43-61). Falmer.

Eris, O., Martelaro, N., \& Badke-Schaub, P. (2014). A comparative analysis of multimodal communication during design sketching in co-located and distributed environments. Design Studies, 35(6), 559-592.

Gero, J. S. (1990). Design prototypes. AI Magazine, 11(4), 16-36.

Gero, J. S. (2000). Computational models of innovative and creative design processes. Technological Forecasting and Social Change, 64, 183-196.

Goel, V. (1995). Sketches of thought. MIT Press.

Goldschmidt, G. (1991). The dialectics of sketching. Creativity Research Journal, 4(2), 123-143.

Goldschmidt, G. (1995). The designer as a team of one. Design Studies, 16, 189-209.

Goldschmidt, G. (2011). Not from scratch. In T. Taura \& Y. Nagai (Eds.), Design creativity 2010. London: Springer.

Goldschmidt, G. (2014). Linkography. MIT Press.

Goldschmidt, G. (2016). Linkographic evidence for concurrent divergent and convergent thinking in creative design. Creativity Research Journal, 28(2), 115-122.

Hammer, D., \& Berland, L. K. (2014). Confusing claims for data. Journal of the Learning Sciences, 23(1), $37-46$.

Hannula, M.S. (2016). The eye on the mathematics. In M. Lepik (Ed.), Proceedings of the 17th International Conference, (pp. 43-57).

Hannula, M.S. \& Williams, G. (2016). Silent gazing during geometry problem solving, insights from eye tracking. In C. Csíkos, A. Rausch \& J. Szitányi (Eds.), Proceedings of the 40th Conference of the International Group for the Psychology of Mathematics Education, Volume 2, (pp. 353-360).

Härkki, T., Seitamaa-Hakkarainen, P. \& Hakkarainen, K. (2018). Line by line, part by part: collaborative sketching for designing.International Journal of Technology and Design Education, 28(2), 471-494

Hatcher, G., Ion, W., Maclachlan, R., Marlow, M., Simpson, B., Wilson, N. \& Wodehouse, A. (2018). Using Linkography to compare creative methods for group ideation. Design Studies, online first. 
Hayhoe, M., \& Ballard, D. (2014). Modeling task control of eye movements minireview. Current Biology, 24, R622-R628.

Hennessy, S., \& Murphy, P. (1999). The potential for collaborative problem solving in design and technology. International Journal of Technology and Design Education, 9(1), 1-36.

Holmqvist, K., Nyström, M., Andersson, R., Dewhurst, R., Jarodzka, H., \& De Weijer, J. (2011). Eye tracking: A comprehensive guide to methods and measures. Oxford University.

Hua, M. (2019). The roles of sketching in supportive creative design. The Design Journal, 22(6), 895-904.

Huang, P.-S. (2017). An exploratory study on remote associates problem solving. Thinking Skills and Creativity, 24, 63-72.

Hultén, M., Artman, H., \& House, D. (2018). A model to analyse students' cooperative idea generation in conceptual design. International Journal of Technology and Design Education, 28(2), 451-470.

Just, M. A., \& Carpenter, P. A. (1980). A theory of reading: From eye fixations to comprehension. Psychological Review, 87, 329-354.

Kälviäinen, M. \& Nylander, N. (2019). Learning Design Process for Sense-Making. In Cumulus Conference Proceedings, Rovaniemi 2019, (pp. 316-333).

Kan, J.W.T. \& Gero, J.S. (2011). Learning to collaborate during team designing. International Conference on Research into Design, Bangalore, Jan 2011. Downloaded from https://www.researchgate.net/publi cation/242234303_Learning_to_Collaborate_During_Team_Designing_Some_Preliminary_Results_ from_Measurement-Based_Tools

Kiernan, L., Ledwith, A., \& Lynch, R. (2020). Comparing the dialogue of experts and novices in interdisciplinary teams to inform design education. International Journal of Technology and Design Education, 30(1), 187-206.

Kleinsmann, M., Deken, F., Dong, A., \& Lauche, K. (2012). Development of design collaboration skills. Journal of Engineering Design, 23(7), 485-506.

Krippendorff, K. (2011). Agreement and informatin in the reliability of coding. Communication Methods and Measures, 5(2), 93-112.

Kvan, T. (2000). Collaborative design: What is it? Automation in Construction, 9, 409-415.

Le Bail, C., Baker, M. \& Détienne, F. (2020). Values and argumentation in collaborative design. CoDesign, latest articles.

Lukander, K., Jagadeesan, S., Chi, H. \& Müller, K. (2013). OMG! - A new robust, wearable and affordable open source Mobile Gaze tracker. In Proceedings of the 15th International Conference on HumanComputer Interaction with Mobile Devices and Services, (pp. 408-11). ACM.

Lyle, J. (2003). Stimulated Recall: A report on its use in naturalistic research. British Educational Research Journal, 29(6), 861-878.

Murphy, K. M. (2012). Transmodality and temporality in design interactions. Journal of Pragmatics, 44, 1966-1981.

Park, H., Lee, S., Lee, M., Chang, M.-S., \& Kwak, H.-W. (2016). Using eye movement data to infer human behavioral intentions. Computers in Human Behavior, 63, 796-804.

Perry, G. T., \& Krippendorff, K. (2013). On the reliability of identifying design moves in protocol analysis. Design Studies, 34, 612-635.

Poole, A. \& Ball, L.J. (2005). Eye tracking in human-computer interaction and usability research. In C. Ghaoui (Ed.), Encyclopedia of human-computer interaction (pp. 211-224). Pennsylvania: Idea Group.

Pourmohamadi, M. \& Gero, J.S. (2011). Linkographer: An analysis tool to study design protocols based on FBS coding scheme. In Proceedings of the 18th International Conference on Engineering Design (ICED'11), Denmark.

Purcell, A. T., \& Gero, J. S. (1998). Drawings and the design process. Design Studies, 19, 389-430.

Rayner, K. (1998). Eye movements in reading and information processing: 20 years of research. Psychological Bulletin, 124(3), 372-422.

Reiter-Palmond, R. \& Leone, S. (2018). Facilitating creativity in interdisciplinary design teams using cognitive processes: A review. Journal of Mechanical Engineering Science, 233(2), 385-394.

Roschelle, J., \& Tiesley, S. D. (1995). The construction of shared knowledge in collaborative problem solving. In C. S. C. Learning (Ed.), CO'Malley (pp. 69-97). Springer.

Safin, S., Détienne, F., Burkhardt, J.-M., Hébert, M. \& Leclercq, P. (2019). The interplay between quality of collaboration, design project evolution and outcome in an architectural design studio. CoDesign, latest articles.

Sauder, J., \& Jin, Y. (2016). A qualitative study of collaborative stimulation in group design thinking. Design Science, 2, e4.

Schembri, M., Farrugia, P., Wodehouse, A. J., Grierson, H., \& Kovacevic, A. (2015). Influence of sketch types on distributed design team work. CoDesign, 12(2), 99-118. 
Schneider, B., Sharma, K., Cuendet, S., Zufferey, G., Dillenbourg, P., \& Pea, R. (2018). Leveraging mobile eye-trackers to capture joint visual attention in co-located collaborative learning groups. International Journal of Computer-Supported Collaborative Learning, 13(3), 241-261.

Smith, K. M. (2015). Conditions influencing the development of design expertise. Design Studies, 36, 77-98.

Staudte, M., \& Crocker, M. W. (2018). On the role of gaze for successful and efficient communication. In G. Brône \& B. Oben (Eds.), Eye-tracking in Interaction (pp. 91-106). John Benjamins.

Sun, L., Xiang, W., Chai, C., Yang, Z., \& Zhang, K. (2014). Designers' perception during sketching. Design Studies, 35, 593-613.

Suwa, M., \& Tversky, B. (1997). What do architects and students perceive in their design sketches? Design Studies, 18, 385-403.

Tang, H. H., Lee, Y. Y., \& Gero, J. S. (2011). Comparing collaborative co-located and distributed design processes in digital and traditional sketching environments. Design Studies, 32, 1-29.

Tessier, V. (2020). Insights on collaborative design research: A scoping review. The Design Journal, 23(5), 655-675.

Thayer, A. L., Petruzelli, A., \& McClurg, C. E. (2018). Addressing the paradox of the team innovation process. American Psychologist, 73(4), 363-375.

Toivanen, M., Lukander, K., \& Puolanmäki, K. (2017). Probabilistic approach to robust wearable gaze tracking. Journal of Eye Movement Research, 10(4), article 2.

Tucker, R., Abbasi, N., Thorpe, G., Ostwald, M., Williams, A., \& Wallis, L. (2014). Enhancing and assessing group and team learning in architecture and related design context. Office for Learning and Teaching, Department of Education.

Ullman, D. G., Wood, S., \& Craig, D. (1990). The importance of drawing in the mechanical design process. Computers \& Graphics, 14(2), 263-270.

Uziak, J., \& Fang, N. (2018). Improving students' freehand sketching skills in mechanical engineering curriculum. International Journal of Mechanical Engineering Education, 46(3), 274-286.

van der Lugt, R. (2005). How sketching can affect the idea generation process in design group meetings. Design Studies, 26, 101-122.

Visser, V. (2006). The cognitive artifacts of designing. Lawrence Erlbaum.

Wiltschnig, S., Christensen, B. T., \& Ball, L. J. (2013). Collaborative problem-solution co-evolution in creative design. Design Studies, 34, 515-542.

Yin, R. K. (1989). Case study research. Sage.

Publisher's Note Springer Nature remains neutral with regard to jurisdictional claims in published maps and institutional affiliations. 